\title{
Tandem RCM of Dienynes for the Construction of Taxol type Carbocyclic Systems
}

Rebeca García-Fandiño, Eva M. Codesido,

Eduardo Sobarzo Sánchez, Luis Castedo, Juan R.

Granja*

Departamento de Química Orgánica e Unidade Asociada ó

C.S.I.C, Facultade de Química, Universidade de Santiago. 15782

Santiago de Compostela, SPAIN.

qojuangg@usc.es

SUPPORTING INFORMATION I 
Methyl (4E, $Z$ )-hex-4-enoate. 4-Hexenoic acid (3.00 g, $26.28 \mathrm{mmol})$ and MeI $(5.0 \mathrm{~mL}, 28.9 \mathrm{mmol})$ were successively added to a suspension of $\mathrm{K}_{2} \mathrm{CO}_{3}(5.40 \mathrm{~g}$, $39.07 \mathrm{mmol})$ in DMF $(15 \mathrm{~mL})$. After stirring for $2 \mathrm{~h}$ at $\mathrm{rt}$, the mixture was poured into water and extracted with $\mathrm{Et}_{2} \mathrm{O}(2 \times 10 \mathrm{~mL})$. The organic phases were washed with water $(3 \times 10 \mathrm{~mL})$, dried over $\mathrm{Na}_{2} \mathrm{SO}_{4}$, filtered and concentrated under reduced pressure. The crude residue was distilled $\left(50{ }^{\circ} \mathrm{C}\right.$ at $\left.1 \mathrm{mmHg}\right)$ to afford $2.60 \mathrm{~g} \mathrm{of}$ wished ester (77\%, colorless oil). ${ }^{1} \mathbf{H}-\mathbf{N M R}\left(\mathrm{CDCl}_{3}, 250 \mathrm{MHz}, \delta\right): 5.44(2 \mathrm{H}, \mathrm{m}, \mathrm{H}-4$, $\mathrm{H}-5), 3.66\left(3 \mathrm{H}, \mathrm{s}, \mathrm{OCH}_{3}\right), 2.33$ (4H, m, H-2, H-3), $1.64(3 \mathrm{H}, \mathrm{d}, J=4.9 \mathrm{~Hz}, \mathrm{H}-6) .{ }^{13} \mathrm{C}-$ NMR $\left(\mathrm{CDCl}_{3}, 63 \mathrm{MHz}, \delta\right): 173.0(\mathrm{CO}), 128.9$ (trans $\left.\mathrm{CH}\right), 128.0$ (cis $\left.\mathrm{CH}\right), 125.7$ (trans $\mathrm{CH}), 124.9$ (cis $\mathrm{CH}), 50.9\left(\mathrm{CH}_{3}\right), 33.6\left(\mathrm{CH}_{2}\right), 27.5\left(\mathrm{CH}_{2}\right), 22.0\left(\right.$ cis $\left.\mathrm{CH}_{3}\right), 17.4$ (trans $\left.\mathrm{CH}_{3}\right)$. EM-IQ ${ }^{+}(\mathrm{m} / \mathrm{z}, \mathrm{I}): 128\left(\mathrm{M}^{+}, 11\right), 97\left(\mathrm{M}^{+}-\mathrm{OMe}, 6\right), 69\left(\mathrm{M}^{+}-\mathrm{CO}_{2} \mathrm{Me}, 11\right)$. HRMS calcd. for $\mathrm{C}_{7} \mathrm{H}_{12} \mathrm{O}_{2}\left(\mathrm{M}^{+}\right): 128.083730$, found 128.083757 .

Methyl (4E, Z)-2-(prop-2-ynyl)hex-4-enoate (4). A solution of methyl (4E, Z)-hex-4-enoate $(3.09 \mathrm{~g}, 24.11 \mathrm{mmol})$ in THF $(50 \mathrm{~mL})$ was added to a solution of LDA in THF (1M, $26.6 \mathrm{~mL}, 26.6 \mathrm{mmol})$ cooled at $-60^{\circ} \mathrm{C}$. After stirring the mixture at that temperature for $30 \mathrm{~min}$, propargyl bromide $(80 \% \mathrm{wt}$. solution in toluene, 4.6 $\mathrm{mL}, 41.0 \mathrm{mmol}$ ) was added to the reaction. After being stirred at $-60{ }^{\circ} \mathrm{C}$ for $45 \mathrm{~min}$, the reaction mixture was poured into $\mathrm{NH}_{4} \mathrm{Cl}(20 \mathrm{~mL})$ and extracted with $\mathrm{Et}_{2} \mathrm{O}(2 \times 10$ $\mathrm{mL})$. The organic phases were washed with $\mathrm{HCl}(10 \%, 10 \mathrm{~mL}), \mathrm{NaHCO}_{3}(10 \mathrm{~mL})$, dried over $\mathrm{Na}_{2} \mathrm{SO}_{4}$, filtered and concentrated under reduced pressure. The crude was purified by flash chromatography on silica gel (5\% EtOAc/hexanes) to afford $1.72 \mathrm{~g}$ of $4\left[43 \%, \mathrm{R}_{\mathrm{f}}=0.5\left(10 \%\right.\right.$ EtOAc/hexanes), colorless oil]. ${ }^{1} \mathbf{H}-\mathbf{N M R}\left(\mathrm{CDCl}_{3}, 250\right.$ $\mathrm{MHz}, \delta): 5.38(2 \mathrm{H}, \mathrm{m}, \mathrm{H}-4, \mathrm{H}-5), 3.69\left(3 \mathrm{H}, \mathrm{s}, \mathrm{OCH}_{3}\right), 3.60(1 \mathrm{H}, \mathrm{m}, \mathrm{H}-2), 2.37$ (4H, $\left.\mathrm{m}, \mathrm{H}-3,-\mathrm{CH}_{2}-\mathrm{C} \equiv\right), 1.96(1 \mathrm{H}, \mathrm{t}, J=2.6 \mathrm{~Hz}, \mathrm{H}-\mathrm{C} \equiv), 1.62(3 \mathrm{H}, \mathrm{m}, \mathrm{H}-6) .{ }^{13} \mathrm{C}-\mathbf{N M R}$ $\left(\mathrm{CDCl}_{3}, 63 \mathrm{MHz}, \delta\right): 174.3(\mathrm{CO}), 128.4(\mathrm{CH}), 126.6(\mathrm{CH}), 81.4(\mathrm{C} \equiv), 70.0(\mathrm{C} \equiv), 51.7$ $\left(\mathrm{CH}_{3}\right), 44.4(\mathrm{CH}), 34.0\left(\mathrm{CH}_{2}\right), 28.2\left(\mathrm{CH}_{2}\right), 17.9\left(\mathrm{CH}_{3}\right) . \mathbf{E M}-\mathrm{IQ}^{+}(\mathrm{m} / \mathrm{z}, \mathrm{I}): 167\left(\mathrm{MH}^{+}\right.$, 8), $136\left(\mathrm{MH}^{+}-\mathrm{OMe}, 3\right), 108\left(\mathrm{MH}^{+}-\mathrm{CO}_{2} \mathrm{Me}, 9\right)$. HRMS calcd. for $\mathrm{C}_{10} \mathrm{H}_{15} \mathrm{O}_{2}$ $\left(\mathrm{MH}^{+}\right): 167.107205$, found 167.107033 .

Triethyl 3-butyne-1,1,1-tricarboxylate. A solution of NaOEt (1.93 g, 28.42 mmol) in EtOH $(24 \mathrm{~mL})$ was added to a solution of triethyl methanetricarboxylate $(6.00 \mathrm{~g}, 25.84 \mathrm{mmol})$ in $\mathrm{Et}_{2} \mathrm{O}(20 \mathrm{~mL})$ cooled in an ice bath. The white sodium salt that precipitated was collected, washed with $\mathrm{Et}_{2} \mathrm{O}$, and dried in vacuo to afford $5.78 \mathrm{~g}$ of triethyl sodiomethanetricarboxylate as a white powder. The resulting salt (5.78 g, $22.73 \mathrm{mmol})$ was dissolved in a 1:1 mixture toluene: DMF $(50 \mathrm{~mL})$ and then treated with propargyl bromide ( $80 \%$ wt. solution in toluene, $5.1 \mathrm{~mL}, 45.5 \mathrm{mmol}$ ). The mixture was stirred and heated at $80{ }^{\circ} \mathrm{C}$ for $1.5 \mathrm{~h}$, cooled, at last filtered and the residue washed with toluene $(3 \times 5 \mathrm{~mL})$. The combinated filtrates were washed with water $(4 \times 15 \mathrm{~mL})$, dried over $\mathrm{Na}_{2} \mathrm{SO}_{4}$, filtered and concentrated under reduced pressure. The crude was distilled $\left(97^{\circ} \mathrm{C}\right.$ at $\left.0.2 \mathrm{mmHg}\right)$ to afford $6.03 \mathrm{~g}$ of triethyl 3butyne-1,1,1-tricarboxylate [ $86 \%, \mathrm{R}_{\mathrm{f}}=0.2(10 \%$ EtOAc/hexanes), pale yellow oil]. ${ }^{1} \mathbf{H}-\mathbf{N M R}\left(\mathrm{CDCl}_{3}, 300 \mathrm{MHz}, \delta\right): 4.25\left(6 \mathrm{H}, \mathrm{q}, J=7.1 \mathrm{~Hz},-\mathrm{OCH}_{2}-\mathrm{Me}\right), 2.98(2 \mathrm{H}, \mathrm{d}, J=$ $2.6 \mathrm{~Hz}, \mathrm{H}-2), 2.01(1 \mathrm{H}, \mathrm{t}, J=2.6 \mathrm{~Hz}, \mathrm{H}-\mathrm{C} \equiv), 1.26\left(9 \mathrm{H}, \mathrm{t}, J=7.1 \mathrm{~Hz},-\mathrm{OCH}_{2} \mathrm{C}_{3}\right)$. ${ }^{13}$ C-NMR $\left(\mathrm{CDCl}_{3}, 75 \mathrm{MHz}, \delta\right): 165.6(3 \mathrm{CO}), 78.6(\mathrm{C} \equiv), 70.6(\mathrm{C} \equiv), 64.4(\mathrm{C}), 62.4$ (3 $\left.\mathrm{CH}_{2}\right), 23.1\left(\mathrm{CH}_{2}\right), 13.75\left(3 \mathrm{CH}_{3}\right) . \mathbf{E M}-\mathrm{IQ}^{+}(\mathrm{m} / \mathrm{z}, \mathrm{I}): 271\left(\mathrm{MH}^{+}, 100\right), 197(35), 125$ (24); HRMS calcd. for $\mathrm{C}_{13} \mathrm{H}_{19} \mathrm{O}_{6}\left(\mathrm{MH}^{+}\right)$: 271.118164, found 271.118680 . 
Representative preparation of diethyl malonates. Preparation of $16 \mathrm{~b}$. A solution of triethyl 3-butyne-1,1,1-tricarboxylate $(3.00 \mathrm{~g}, 11.10 \mathrm{mmol})$ in THF $(3 \mathrm{~mL})$ was added via cannula to a suspension of sodium ethoxide (980 mg, $14.4 \mathrm{mmol})$ in THF (35 mL). After being stirred at $\mathrm{rt}$ for $1.5 \mathrm{~h}$, the alkylating agent (2 equiv., 22.2 mmol) was added and the mixture stirred for $3 \mathrm{~h}$, poured into brine $(30 \mathrm{~mL})$ and extracted with $\mathrm{Et}_{2} \mathrm{O}(2 \times 20 \mathrm{~mL})$. The combined organic phases were dried over $\mathrm{Na}_{2} \mathrm{SO}_{4}$, filtered and concentrated under reduced pressure. The crude was purified by flash chromatography on silica gel (4\% EtOAc/hexanes) to afford $1.03 \mathrm{~g}$ of $\mathbf{1 6 b}$ $\left[35 \%, \mathrm{R}_{\mathrm{f}}=0.4(10 \% \mathrm{EtOAc} / \mathrm{hexanes})\right.$, yellow oil]. ${ }^{1} \mathbf{H}-\mathbf{N M R}\left(\mathrm{CDCl}_{3}, 250 \mathrm{MHz}, \delta\right)$ : $5.48(1 \mathrm{H}, \mathrm{m}, \mathrm{H}-\mathrm{C}=), 4.97(1 \mathrm{H}, \mathrm{m}, \mathrm{H}-\mathrm{C}=), 4.11\left(4 \mathrm{H}, \mathrm{m}, \mathrm{CO}_{2} \mathrm{C}_{\underline{H}}\right), 2.70\left(4 \mathrm{H}, \mathrm{m},-\mathrm{C}_{{ }_{2}}-\right.$ $\left.\mathrm{C} \equiv,-\mathrm{C} \underline{H}_{2}-\mathrm{C}=\right), 2.07\left(2 \mathrm{H}, \mathrm{m}, \mathrm{CH}_{3}-\underline{\mathrm{C}}_{2}-\mathrm{C}=\right), 1.94(1 \mathrm{H}, \mathrm{t}, J=2.7 \mathrm{~Hz}, \mathrm{H}-\mathrm{C} \equiv), 1.18(6 \mathrm{H}$, $\left.\mathrm{t}, J=7.2 \mathrm{~Hz}, \mathrm{CO}_{2} \mathrm{CH}_{2} \underline{C H}_{3}\right), 0.88\left(3 \mathrm{H}, \mathrm{t}, J=7.5 \mathrm{~Hz}, \underline{\mathrm{C}}_{3}-\mathrm{CH}_{2}-\mathrm{C}=\right) .{ }^{13} \mathbf{C}-\mathbf{N M R}$ $\left(\mathrm{CDCl}_{3}, 63 \mathrm{MHz}, \delta\right): 169.7(2 \mathrm{CO}), 136.4(\mathrm{CH}), 121.6(\mathrm{CH}), 78.9(\mathrm{C} \equiv), 71.1(\mathrm{C} \equiv)$, $61.4\left(\mathrm{CH}_{2}\right), 61.1\left(\mathrm{CH}_{2}\right), 56.5(\mathrm{C}), 29.5\left(\mathrm{CH}_{2}\right), 22.2\left(\mathrm{CH}_{2}\right), 20.5\left(\mathrm{CH}_{2}\right), 14.0\left(\mathrm{CH}_{3}\right)$, $13.9\left(\mathrm{CH}_{3}\right), 13.6\left(\mathrm{CH}_{3}\right)$; EM-IQ ${ }^{+}(\mathrm{m} / \mathrm{z}, \mathrm{I}): 267\left(\mathrm{MH}^{+}, 100\right), 194\left(\mathrm{MH}^{+}-\mathrm{CO}_{2} \mathrm{Et}, 5\right), 121$ $\left(\mathrm{MH}^{+}-\left(\mathrm{CO}_{2} \mathrm{Et}\right)_{2}, 5\right)$; HRMS calcd. for $\mathrm{C}_{15} \mathrm{H}_{23} \mathrm{O}_{4}\left(\mathrm{MH}^{+}\right)$: 267.159634, found 267.160073.

Preparation of 16c. Following the above described procedure, compound 16c was obtained in $88 \%$. [ $\mathrm{R}_{\mathrm{f}}=0.5(15 \%$ EtOAc/hexanes $)$, yellow oil $] .{ }^{1} \mathbf{H}-\mathbf{N M R}\left(\mathrm{CDCl}_{3}\right.$, $300 \mathrm{MHz}, \delta): 5.52\left(1 \mathrm{H}, \mathrm{dd}, J=15.2\right.$ and $\left.6.9 \mathrm{~Hz},{ }^{\mathrm{i}} \mathrm{Pr}-\mathrm{C} \underline{H}=\mathrm{CHR}\right), 5.15\left(1 \mathrm{H}, \mathrm{m},{ }^{\mathrm{i}} \mathrm{Pr}-\right.$ $\mathrm{CH}=\mathrm{C} \underline{H R}), 4.17\left(4 \mathrm{H}, \mathrm{q}, J=7.1 \mathrm{~Hz}, \mathrm{CO}_{2} \underline{\mathrm{CH}}_{2}\right), 2.75\left(2 \mathrm{H}, \mathrm{d}, J=2.6 \mathrm{~Hz},-\mathrm{C}_{2}-\mathrm{C} \equiv\right)$, $2.70\left(2 \mathrm{H}, \mathrm{d}, J=7.5 \mathrm{~Hz},-\underline{C}_{2}-\mathrm{C}=\right), 2.21\left(1 \mathrm{H}, \mathrm{m}, \mathrm{Me}_{2} \mathrm{C} \underline{H}\right), 1.98(1 \mathrm{H}, \mathrm{t}, J=2.6 \mathrm{~Hz}, \mathrm{H}-$ $\mathrm{C} \equiv), 1.23\left(6 \mathrm{H}, \mathrm{t}, J=7.1 \mathrm{~Hz}, \mathrm{CO}_{2} \mathrm{CH}_{2} \underline{\mathrm{C}}_{3}\right), 0.92(6 \mathrm{H}, \mathrm{d}, J=6.8 \mathrm{~Hz}, \mathrm{Me}) .{ }^{13} \mathrm{C}-\mathbf{N M R}$ $\left(\mathrm{CDCl}_{3}, 75 \mathrm{MHz}, \delta\right): 169.8(2 \mathrm{CO}), 143.2(\mathrm{CH}), 119.7(\mathrm{CH}), 79.1(\mathrm{C} \equiv), 71.2(\mathrm{C} \equiv)$, $61.5\left(2 \mathrm{CH}_{2}\right), 57.0(\mathrm{C}), 35.0\left(\mathrm{CH}_{2}\right), 31.1(\mathrm{CH}), 22.0\left(2 \mathrm{CH}_{3}\right), 21.7\left(\mathrm{CH}_{2}\right), 14.1(2$ $\left.\mathrm{CH}_{3}\right)$. EM-IQ ${ }^{+}(\mathrm{m} / \mathrm{z}, \mathrm{I}): 281\left(\mathrm{MH}^{+}, 58\right), 207$ (25), 133 (17). HRMS calcd. for $\mathrm{C}_{16} \mathrm{H}_{25} \mathrm{O}_{4}\left(\mathrm{MH}^{+}\right): 281.175285$, found 281.175641 .

Preparation of 16d. Following the above described procedure, compound 16d was obtained in $75 \%$. [ $\mathrm{R}_{\mathrm{f}}=0.4(15 \%$ EtOAc/hexanes), yellow oil $]$ H-NMR $\left(\mathrm{CDCl}_{3}\right.$, $300 \mathrm{MHz}, \delta): 4.89(1 \mathrm{H}, \mathrm{t}, J=1.4 \mathrm{~Hz}, \mathrm{H}-\mathrm{C}=), 4.17\left(4 \mathrm{H}, \mathrm{m}, \mathrm{CO}_{2}-\mathrm{C}_{2}\right), 2.75(4 \mathrm{H}, \mathrm{m},-$ $\left.\mathrm{C} \underline{H}_{2}-\mathrm{C}=,-\underline{C H}_{2}-\mathrm{C} \equiv\right), 1.97(1 \mathrm{H}, \mathrm{t}, J=2.7 \mathrm{~Hz}, \mathrm{H}-\mathrm{C} \equiv), 1.65(3 \mathrm{H}, \mathrm{s}, \mathrm{Me}-\mathrm{C}=), 1.64(3 \mathrm{H}, \mathrm{s}$, $\mathrm{Me}-\mathrm{C}=), 1.23\left(6 \mathrm{H}, \mathrm{t}, J=7.1 \mathrm{~Hz}, \mathrm{CO}_{2}-\mathrm{CH}_{2}-\underline{C H}_{3}\right) .{ }^{13} \mathbf{C}-\mathbf{N M R}\left(\mathrm{CDCl}_{3}, 75 \mathrm{MHz}, \delta\right)$ : $170.0(2 \mathrm{CO}), 136.6(\mathrm{C}), 117.1(\mathrm{CH}), 71.1(\mathrm{C} \equiv), 79.4(\mathrm{C} \equiv), 61.5\left(2 \mathrm{CH}_{2}\right), 57.0(\mathrm{C})$, $30.6\left(\mathrm{CH}_{2}\right), 26.0\left(\mathrm{CH}_{3}\right), 22.4\left(\mathrm{CH}_{2}\right), 18.0\left(\mathrm{CH}_{3}\right), 14.0\left(2 \mathrm{CH}_{3}\right)$. EM-IQ ${ }^{+}(\mathrm{m} / \mathrm{z}, \mathrm{I}): 267$ $\left(\mathrm{MH}^{+}, 1\right), 205$ (5). HRMS calcd. for $\mathrm{C}_{15} \mathrm{H}_{23} \mathrm{O}_{4}\left(\mathrm{MH}^{+}\right)$: 267.159634, found 267.158819 .

Representative malonate decarboxilation. Preparation of Ethyl (4Z)-2(prop-2-ynyl)hept-4-enoate. Malonate 16b (3.19 g, $12.01 \mathrm{mmol})$ was added to a solution of NaOEt $(1.23 \mathrm{~g}, 18.07 \mathrm{mmol})$ in EtOH $(50 \mathrm{~mL})$. The reaction mixture was refluxed for 2 days, allowed to come to rt, poured into $\mathrm{NaCl}(40 \mathrm{~mL})$ and then treated with $\mathrm{HCl}(10 \%, 20 \mathrm{~mL})$. The aqueous layer was extracted with $\mathrm{Et}_{2} \mathrm{O}(3 \times 20 \mathrm{~mL})$ and the combined organic phases were dried over $\mathrm{Na}_{2} \mathrm{SO}_{4}$, filtered and concentrated under reduced pressure. The crude was purified by flash chromatography on silica gel $(1 \%$ EtOAc/hexanes) to yield $1.28 \mathrm{~g}$ of the whised ethyl ester. [55\%, $\mathrm{R}_{\mathrm{f}}=0.6(10 \%$ EtOAc/hexanes), yellow oil]. ${ }^{1} \mathbf{H}-\mathbf{N M R}\left(\mathrm{CDCl}_{3}, 250 \mathrm{MHz}, \delta\right): 5.44(1 \mathrm{H}, \mathrm{m}, \mathrm{H}-\mathrm{C}=)$, $5.19(1 \mathrm{H}, \mathrm{m}, \mathrm{H}-\mathrm{C}=), 4.11\left(2 \mathrm{H}, \mathrm{q}, J=7.1 \mathrm{~Hz},-\mathrm{CO}_{2}-\underline{\mathrm{C}}_{2}-\right), 2.51(1 \mathrm{H}, \mathrm{m}, \mathrm{H}-2), 2.35$ $\left(4 \mathrm{H}, \mathrm{m}, \mathrm{H}-3, \mathrm{CH}_{2}-\mathrm{C} \equiv\right), 2.01(3 \mathrm{H}, \mathrm{m}, \mathrm{H}-6, \mathrm{H}-\mathrm{C} \equiv), 1.21\left(3 \mathrm{H}, \mathrm{t}, J=7.1 \mathrm{~Hz},-\mathrm{CO}_{2}-\mathrm{CH}_{2}-\right.$ 
$\left.\mathrm{C}_{3}\right), 0.90(3 \mathrm{H}, \mathrm{t}, J=7.5 \mathrm{~Hz}, \mathrm{H}-7) .{ }^{13} \mathrm{C}-\mathrm{NMR}\left(\mathrm{CDCl}_{3}, 63 \mathrm{MHz}, \delta\right): 173.8(\mathrm{CO})$, $134.6(\mathrm{CH}), 124.3(\mathrm{CH}), 81.4(\mathrm{C} \equiv), 69.8(\mathrm{C} \equiv), 60.5\left(\mathrm{CH}_{2}\right), 44.4(\mathrm{CH}), 28.5\left(\mathrm{CH}_{2}\right)$, $20.5\left(\mathrm{CH}_{2}\right), 20.3\left(\mathrm{CH}_{2}\right), 14.2\left(\mathrm{CH}_{3}\right), 14.1\left(\mathrm{CH}_{3}\right) . \mathbf{E M}-\mathrm{IQ}^{+}(\mathrm{m} / \mathrm{z}, \mathrm{I}): 195\left(\mathrm{MH}^{+}, 53\right)$, 167 (27), 149 (17), 121 (100). HRMS calcd. for $\mathrm{C}_{12} \mathrm{H}_{19} \mathrm{O}_{2}\left(\mathrm{MH}^{+}\right)$: 195.138505, found 195.138842 .

Preparation of Ethyl (4E)-6-methyl-2-(prop-2-ynyl)hept-4-enoate. Following the above described procedure, Ethyl (4E)-6-methyl-2-(prop-2-ynyl)hept4-enoate was obtained in $50 \%$. [ $\mathrm{R}_{\mathrm{f}}=0.5(15 \%$ EtOAc/hexanes $)$, yellow oil $]{ }^{1} \mathbf{H}-\mathbf{N M R}$ $\left(\mathrm{CDCl}_{3}, 250 \mathrm{MHz}, \delta\right): 5.47(1 \mathrm{H}, \mathrm{dd}, J=15.5$ and $6.3 \mathrm{~Hz}, \mathrm{H}-5), 5.26(1 \mathrm{H}, \mathrm{m}, \mathrm{H}-4)$, $4.15\left(2 \mathrm{H}, \mathrm{q}, J=7.1 \mathrm{~Hz},-\mathrm{CO}_{2}-\underline{\mathrm{C}}_{2}{ }^{-}\right), 2.56(1 \mathrm{H}, \mathrm{m}, \mathrm{H}-6), 2.33\left(5 \mathrm{H}, \mathrm{m}, \mathrm{H}-3, \mathrm{H}-2, \mathrm{C}_{2}{ }^{-}\right.$ $\mathrm{C} \equiv), 1.98(1 \mathrm{H}, \mathrm{t}, J=2.6 \mathrm{~Hz}, \mathrm{H}-\mathrm{C} \equiv), 1.26\left(3 \mathrm{H}, \mathrm{t}, J=7.1 \mathrm{~Hz},-\mathrm{CO}_{2}-\mathrm{CH}_{2}-\mathrm{C}_{3}\right), 0.94$ $(6 \mathrm{H}, \mathrm{d}, J=6.8 \mathrm{~Hz}, \mathrm{Me}) ;{ }^{13} \mathbf{C}-\mathbf{N M R}\left(\mathrm{CDCl}_{3}, 63 \mathrm{MHz}, \delta\right): 173.5(\mathrm{CO}), 140.7(\mathrm{CH})$, $122.3(\mathrm{CH}), 81.2(\mathrm{C} \equiv), 69.6(\mathrm{C} \equiv), 60.1\left(\mathrm{CH}_{2}\right), 44.3(\mathrm{CH}), 33.8\left(\mathrm{CH}_{2}\right), 30.8(\mathrm{CH}), 22.2$ $\left(2 \mathrm{CH}_{3}\right), 19.9\left(\mathrm{CH}_{2}\right), 14.0\left(\mathrm{CH}_{3}\right) ; \mathbf{E M}-\mathrm{IQ}^{+}(\mathrm{m} / \mathrm{z}, \mathrm{I}): 209\left(\mathrm{MH}^{+}, 29\right), 136\left(\mathrm{MH}^{+}-\mathrm{CO}_{2} \mathrm{Et}\right.$, 2); HRMS calcd. for $\mathrm{C}_{13} \mathrm{H}_{21} \mathrm{O}_{2}\left(\mathrm{MH}^{+}\right): 209.154155$, found 209.154326 .

Preparation of Ethyl 5-methyl-2-(prop-2-ynyl)hex-4-enoate. Following the above described procedure Ethyl 5-methyl-2-(prop-2-ynyl)hex-4-enoate was obtained in $32 \%$. $\left[\mathrm{R}_{\mathrm{f}}=0.6(10 \%\right.$ EtOAc/hexanes $)$, yellow oil $] .{ }^{1} \mathbf{H}-\mathbf{N M R}\left(\mathrm{CDCl}_{3}, 300 \mathrm{MHz}, \delta\right)$ : $5.04(1 \mathrm{H}, \mathrm{t}, J=1.4 \mathrm{~Hz}, \mathrm{H}-4), 4.15\left(2 \mathrm{H}, \mathrm{q}, J=7.1 \mathrm{~Hz}, \mathrm{CO}_{2} \underline{\mathrm{C}}_{2}\right), 2.54(1 \mathrm{H}, \mathrm{m}, \mathrm{H}-2)$, $2.39\left(4 \mathrm{H}, \mathrm{m}, \mathrm{H}-3, \mathrm{C}_{2}-\mathrm{C} \equiv\right), 1.96(1 \mathrm{H}, \mathrm{t}, J=2.6 \mathrm{~Hz}, \mathrm{H}-\mathrm{C} \equiv), 1.68(3 \mathrm{H}, \mathrm{s}, \mathrm{Me}-\mathrm{C}=)$, $1.61(3 \mathrm{H}, \mathrm{s}, \mathrm{Me}-\mathrm{C}=), 1.24\left(3 \mathrm{H}, \mathrm{t}, J=7.1 \mathrm{~Hz}, \mathrm{CO}_{2} \mathrm{CH}_{2} \mathrm{CH}_{3}\right) .{ }^{13} \mathbf{C}-\mathbf{N M R}\left(\mathrm{CDCl}_{3}, 75\right.$ $\mathrm{MHz}, \delta): 174.1(\mathrm{CO}), 134.6(\mathrm{C}), 120.1(\mathrm{CH}), 81.7(\mathrm{C} \equiv), 69.7(\mathrm{C} \equiv), 60.5\left(\mathrm{CH}_{2}\right), 44.7$ (CH), $29.6\left(\mathrm{CH}_{2}\right), 25.8\left(\mathrm{CH}_{3}\right), 20.3\left(\mathrm{CH}_{2}\right), 17.8\left(\mathrm{CH}_{3}\right), 14.2\left(\mathrm{CH}_{3}\right)$. EM-IQ ${ }^{+}(\mathrm{m} / \mathrm{z}, \mathrm{I})$ : $195\left(\mathrm{MH}^{+}, 1\right)$.

(4R, 5S)-4-methyl-3-[(4E)-6-methylhept-4-enyl]-5-phenyl-1,3-oxazolidin-2one (17). To a flask containing (4E)-6-methyl-hept-4-enoic acid $(2.32 \mathrm{~g}$ of crude residue) and $\mathrm{Et}_{3} \mathrm{~N}(3.8 \mathrm{~mL}, 27.1 \mathrm{mmol})$ in THF $(8 \mathrm{~mL})$ at $-78{ }^{\circ} \mathrm{C}$ was slowly added pivaloyl chloride $(3.3 \mathrm{~mL}, 27.2 \mathrm{mmol})$. The thick white paste was allowed to stir at 0 ${ }^{\circ} \mathrm{C}$ for $1 \mathrm{~h}$. In a separated flask, (4R, 5S)-(+)-4-Methyl-5-phenyl-2-oxazolidinone (4.37 g, $24.66 \mathrm{mmol})$ was dissolved in THF $(11 \mathrm{~mL})$ at rt. A $10 \%$ mol catalytic amount of DMAP (302 mg, $2.46 \mathrm{mmol}$ ) was added, followed by $\mathrm{Et}_{3} \mathrm{~N}$ (3.4 mL, 24.6 mmol). This solution was then added to the above mixed anhydride at $-78^{\circ} \mathrm{C}$ over 5 min. The mixture was then allowed to stir for $12 \mathrm{~h}$ at $\mathrm{rt}$ to ensure complete reaction. The volatiles were removed in vacuo and the resultant white paste was dissolved in $\mathrm{CH}_{2} \mathrm{Cl}_{2}(11 \mathrm{~mL})$ and washed with $\mathrm{NaOH}(1 \mathrm{M}, 6 \mathrm{~mL})$. The organic phase was washed with brine and then dried, filtered and concentrated under reduced pressure. The crude was purified by flash chromatography on silica gel (12\% EtOAc/hexanes) to yield $7.41 \mathrm{~g}$ of 17 in $80 \%$. [7.41 g, $\mathrm{R}_{\mathrm{f}}=0.75$ (30\% EtOAc/hexanes), pale yellow thick oil]. ${ }^{1}$ H-NMR $\left(\mathrm{CDCl}_{3}, 500 \mathrm{MHz}, \delta\right): 7.36(5 \mathrm{H}, \mathrm{m}, \mathrm{H}-\mathrm{Ar}), 5.65(1 \mathrm{H}, \mathrm{d}, J=7.3 \mathrm{~Hz}, \mathrm{CH}-\mathrm{Ph})$, $5.43(2 \mathrm{H}, \mathrm{m}, \mathrm{H}-\mathrm{C}=), 4.75\left(1 \mathrm{H}, \mathrm{m}, \mathrm{C} \underline{H}-\mathrm{CH}_{3}\right), 3.01\left(2 \mathrm{H}, \mathrm{m}, \mathrm{CH}_{2}\right), 2.35\left(2 \mathrm{H}, \mathrm{m}, \mathrm{CH}_{2}\right)$, $2.22\left(1 \mathrm{H}, \mathrm{m}, \mathrm{C} \underline{\mathrm{H}}\left(\mathrm{CH}_{3}\right)_{2}\right), 0.95(6 \mathrm{H}, \mathrm{d}, J=6.7 \mathrm{~Hz}, \mathrm{Me}), 0.87\left(3 \mathrm{H}, \mathrm{d}, J=6.5 \mathrm{~Hz}, \mathrm{CH}_{3}\right)$. ${ }^{13} \mathbf{C}-\mathbf{N M R}\left(\mathrm{CDCl}_{3}, 125 \mathrm{MHz}, \delta\right): 172.5$ (CO), 153.0 (CO), 133.3 (C Ar), 138.9, 128.7 $(\mathrm{CH}), 128.6(\mathrm{CH}), 125.6(\mathrm{CH}), 124.8(\mathrm{CH}), 78.9(\mathrm{CH}), 54.7(\mathrm{CH}), 35.6\left(\mathrm{CH}_{2}\right), 30.9$ $(\mathrm{CH}), 27.2\left(\mathrm{CH}_{2}\right), 22.4\left(2 \mathrm{CH}_{3}\right), 14.5\left(\mathrm{CH}_{3}\right) . \mathbf{E M}-\mathbf{I Q}^{+}(\mathrm{m} / \mathrm{e}, \mathrm{I}): 302\left(\mathrm{MH}^{+}, 28\right), 206$ (28), 178 (43), 134 (100). HRMS calcd. for $\mathrm{C}_{18} \mathrm{H}_{24} \mathrm{NO}_{3}\left(\mathrm{MH}^{+}\right)$: 302.175619, found 302.176318 .

\footnotetext{
${ }^{1}$ Kaga, H.; Goto, K.; Takahashi, T.; Hino, M.; Tokuhashi, T.; Orito, K. Tetrahedron 1996, 52,84518470 .
} 


\section{Preparation of}

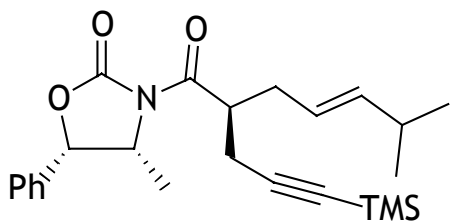

LiHMDS (1M, $4.3 \mathrm{~mL}, 4.3 \mathrm{mmol})$ was added to a solution of compound 17 (1.20 g, $3.99 \mathrm{mmol})$ in THF $(25 \mathrm{~mL})$ cooled at $-78{ }^{\circ} \mathrm{C}$. The reaction mixture was stirred at $0{ }^{\circ} \mathrm{C}$ for $3 \mathrm{~h}$, poured into $\mathrm{NH}_{4} \mathrm{Cl}(30 \mathrm{~mL})$ and then extracted with $\mathrm{CH}_{2} \mathrm{Cl}_{2}(3 \times 15 \mathrm{~mL})$. The combined organic phases were dried over $\mathrm{Na}_{2} \mathrm{SO}_{4}$, filtered and concentrated under reduced pressure. The crude was purified by flash chromatography on silica gel (5\% EtOAc/hexanes) to afford $1.10 \mathrm{~g}$ of the whished product. [67\%, $\mathrm{R}_{\mathrm{f}}=0.6\left(15 \%\right.$ EtOAc/hexanes), yellow oil]. ${ }^{1} \mathbf{H}-$ NMR $\left(\mathrm{CDCl}_{3}, 500 \mathrm{MHz}, \delta\right): 7.36(5 \mathrm{H}, \mathrm{m}, \mathrm{H}-\mathrm{Ar}), 5.61(1 \mathrm{H}, \mathrm{d}, J=7.3 \mathrm{~Hz}, \mathrm{CH}-\mathrm{Ph})$, $5.47(1 \mathrm{H}, \mathrm{dd}, J=15.3$ and $6.6 \mathrm{~Hz}, \mathrm{H}-\mathrm{C}=), 5.32(1 \mathrm{H}, \mathrm{m}, \mathrm{H}-\mathrm{C}=), 4.77(1 \mathrm{H}, \mathrm{m}, \mathrm{C} \underline{H}-$ $\left.\mathrm{CH}_{3}\right), 4.05(1 \mathrm{H}, \mathrm{m}, \mathrm{CO}-\mathrm{CH}), 2.52\left(2 \mathrm{H}, \mathrm{m}, \mathrm{CH}{ }_{2}-\mathrm{C} \equiv\right), 2.38\left(1 \mathrm{H}, \mathrm{m}, \mathrm{C} \underline{\mathrm{H}}\left(\mathrm{CH}_{3}\right)_{2}\right), 2.25$ $\left(2 \mathrm{H}, \mathrm{m}, \mathrm{CH}_{2}-\mathrm{C}=\right), 0.95\left(6 \mathrm{H}, \mathrm{d}, J=6.8 \mathrm{~Hz}, \mathrm{CH}\left(\mathrm{CH}_{3}\right)_{2}\right), 0.89\left(3 \mathrm{H}, \mathrm{d}, J=6.6 \mathrm{~Hz}, \mathrm{CH}_{3}\right)$, 0.08 (9H, s, $\left.\mathrm{Me}_{3} \mathrm{Si}\right) .{ }^{13} \mathrm{C}-\mathrm{NMR}\left(\mathrm{CDCl}_{3}, 75 \mathrm{MHz}, \delta\right): 174.1(\mathrm{CO}), 152.6(\mathrm{CO}), 141.2$ (CH), 133.3 (C Ar), 128.7(CH Ar), 125.6 (CH Ar), 122.6 (CH-C=), $103.9(\mathrm{C} \equiv), 86.1$ $(\mathrm{C} \equiv), 78.8(\mathrm{CH}), 54.9(\mathrm{CH}), 42.5(\mathrm{CH}), 34.6\left(\mathrm{CH}_{2}\right), 31.0(\mathrm{CH}), 22.5\left(2 \mathrm{CH}_{3}\right), 21.7$ $\left(\mathrm{CH}_{2}\right), 14.6\left(\mathrm{CH}_{3}\right), 0.01\left(3 \mathrm{CH}_{3}\right) . \mathbf{E M}-\mathrm{IQ}^{+}(\mathrm{m} / \mathrm{e}, \mathrm{I}): 412\left(\mathrm{MH}^{+}, 10\right), 397(12), 250$ (100). HRMS calcd. for $\mathrm{C}_{24} \mathrm{H}_{34} \mathrm{NO}_{3} \mathrm{Si}\left(\mathrm{MH}^{+}\right)$: 412.230798 , found 412.232037 .

Representative reduction of esters. Preparation of $(4 E, Z)-2-($ prop-2ynyl)-4-hexen-1-ol. $\mathrm{LiAlH}_{4}(0.57 \mathrm{~g}, 15.02 \mathrm{mmol})$ was added to a solution of ethyl (4Z)-2-(prop-2-ynyl)-hept-4-enoate $(1.65 \mathrm{~g}, 9.94 \mathrm{mmol})$ in $\mathrm{Et}_{2} \mathrm{O}(50 \mathrm{~mL})$ at $0{ }^{\circ} \mathrm{C}$. The reaction mixture was stirred at that temperature for $30 \mathrm{~min}$ and quenched by adding $\mathrm{H}_{2} \mathrm{SO}_{4}(5 \%, 40 \mathrm{~mL})$. The mixture was extracted with $\mathrm{Et}_{2} \mathrm{O}(2 \times 20 \mathrm{~mL})$, dried over $\mathrm{Na}_{2} \mathrm{SO}_{4}$, filtered and concentrated under reduced pressure. The crude was purified by flash chromatography on silica gel $(10 \% \mathrm{EtOAc} / \mathrm{h}$ exanes) to afford $1.29 \mathrm{~g}$ of whished alcohol $\left[94 \%, \mathrm{R}_{\mathrm{f}}=0.3\left(10 \%\right.\right.$ EtOAc/hexanes), light yellow oil]. ${ }^{1} \mathbf{H}-\mathbf{N M R}\left(\mathrm{CDCl}_{3}\right.$, $500 \mathrm{MHz}, \delta): 5.50(1 \mathrm{H}, \mathrm{m}, \mathrm{H}-4$ or H-5), $5.37(1 \mathrm{H}, \mathrm{m}, \mathrm{H}-4$ or H-5), $3.64(2 \mathrm{H}, \mathrm{m}, \mathrm{H}-1)$, $1.97(1 \mathrm{H}, \mathrm{t}, J=2.6 \mathrm{~Hz}, \mathrm{H}-\mathrm{C} \equiv), 1.72(3 \mathrm{H}, \mathrm{m}, \mathrm{H}-6) .{ }^{13} \mathbf{C}-\mathbf{N M R}\left(\mathrm{CDCl}_{3}, 125 \mathrm{MHz}, \delta\right)$ : $128.3(\mathrm{CH}), 127.5(\mathrm{CH}), 82.6(\mathrm{C} \equiv), 69.6(\mathrm{C} \equiv), 64.9\left(\mathrm{CH}_{2}\right), 39.9(\mathrm{CH}), 33.6\left(\mathrm{CH}_{2}\right)$, $19.9\left(\mathrm{CH}_{2}\right), 17.9\left(\mathrm{CH}_{3}\right)$; EM-IQ ${ }^{+}(\mathrm{m} / \mathrm{z}, \mathrm{I}): 139\left(\mathrm{MH}^{+}, 5\right), 121\left(\mathrm{MH}^{+}-\mathrm{H}_{2} \mathrm{O}, 15\right) . \mathbf{H R M S}$ calcd. for $\mathrm{C}_{9} \mathrm{H}_{15} \mathrm{O}\left(\mathrm{MH}^{+}\right)$: 139.112290, found 139.112407 .

Preparation of (4Z)-2-(prop-2-ynyl)-4-hepten-1-ol. Following the above described procedure, (4Z)-2-(prop-2-ynyl)-4-hepten-1-ol was obtained in 90\%. $\left[\mathrm{R}_{\mathrm{f}}=0.15\right.$ (15\% EtOAc/hexanes), pale yellow oil]. ${ }^{1} \mathbf{H}-\mathbf{N M R}\left(\mathrm{CDCl}_{3}, 250 \mathrm{MHz}, \delta\right)$ : 5.45 (2H, m, H-4, H-5), 3.61 (2H, m, H-1), 2.25 (2H, m, H-6), $2.13\left(4 \mathrm{H}, \mathrm{m}, \mathrm{C} \underline{H}_{2}-\mathrm{C} \equiv\right.$, H-3), $1.96(1 \mathrm{H}, \mathrm{t}, J=2.7 \mathrm{~Hz}, \mathrm{H}-\mathrm{C} \equiv), 1.75(1 \mathrm{H}, \mathrm{m}, \mathrm{H}-2), 0.93(3 \mathrm{H}, \mathrm{t}, J=7.5 \mathrm{~Hz}, \mathrm{H}-$ 7). ${ }^{13} \mathrm{C}-\mathrm{NMR}\left(\mathrm{CDCl}_{3}, 63 \mathrm{MHz}, \delta\right): 133.9(\mathrm{CH}), 126.0(\mathrm{CH}), 82.5(\mathrm{C} \equiv), 69.7(\mathrm{C} \equiv)$, $64.7\left(\mathrm{CH}_{2}\right), 40.2(\mathrm{CH}), 28.0\left(\mathrm{CH}_{2}\right), 20.6\left(\mathrm{CH}_{2}\right), 19.8\left(\mathrm{CH}_{2}\right), 14.2\left(\mathrm{CH}_{3}\right)$. EM-IQ ${ }^{+}$ $(\mathrm{m} / \mathrm{e}, \mathrm{I}): 153\left(\mathrm{MH}^{+}, 36\right), 135\left(\mathrm{MH}^{+}-\mathrm{H}_{2} \mathrm{O}, 51\right)$. HRMS calcd. for $\mathrm{C}_{10} \mathrm{H}_{17} \mathrm{O}\left(\mathrm{MH}^{+}\right)$: 153.127940, found 153.128589 .

Preparation of (4E)-6-methyl-2-(prop-2-ynyl)-4-hepten-1-ol. Following the above described procedure, (4E)-6-methyl-2-(prop-2-ynyl)-4-hepten-1-ol was obtained in $89 \%$. [ $\left[\mathrm{R}_{\mathrm{f}}=0.3(15 \%\right.$ EtOAc/ hexanes $)$, pale yellow oil]. ${ }^{1} \mathbf{H}-\mathbf{N M R}$ $\left(\mathrm{CDCl}_{3}, 300 \mathrm{MHz}, \delta\right): 5.45(1 \mathrm{H}, \mathrm{dd}, J=15.3$ and $6.4 \mathrm{~Hz}, \mathrm{H}-5), 5.30(1 \mathrm{H}, \mathrm{dt}, J=15.2$ and 7.1 Hz, H-4), $3.62(2 \mathrm{H}, \mathrm{m}, \mathrm{H}-1), 2.24(1 \mathrm{H}, \mathrm{m}, \mathrm{H}-6), 1.96(1 \mathrm{H}, \mathrm{t}, J=2.6 \mathrm{~Hz}, \mathrm{H}-$ $\mathrm{C} \equiv), 1.76(1 \mathrm{H}, \mathrm{m}, \mathrm{H}-2), 0.95(6 \mathrm{H}, \mathrm{d}, J=6.7 \mathrm{~Hz}) .{ }^{13} \mathrm{C}-\mathrm{NMR}\left(\mathrm{CDCl}_{3}, 75 \mathrm{MHz}, \delta\right)$ : $140.3(\mathrm{CH}), 124.0(\mathrm{CH}), 82.6(\mathrm{C} \equiv), 69.7(\mathrm{C} \equiv), 64.8\left(\mathrm{CH}_{2}\right), 40.0(\mathrm{CH}), 33.6\left(\mathrm{CH}_{2}\right)$, 
$31.0(\mathrm{CH}), 22.6\left(2 \mathrm{CH}_{3}\right), 19.8\left(\mathrm{CH}_{2}\right)$. EM-IQ ${ }^{+}(\mathrm{m} / \mathrm{e}, \mathrm{I}): 167\left(\mathrm{MH}^{+}, 31\right), 149\left(\mathrm{MH}^{+}-\right.$ $\left.\mathrm{H}_{2} \mathrm{O}, 51\right)$. HRMS calcd. for $\mathrm{C}_{11} \mathrm{H}_{19} \mathrm{O}\left(\mathrm{MH}^{+}\right)$: 167.143590, found 167.144312.

Preparation of $(2 S, 4 E)-6$-methyl-2-(prop-2-ynyl)-4-hepten-1-ol (19c). Following the above described procedure, (2S, 4E)-6-methyl-2-(prop-2-ynyl)-4hepten-1-ol was obtained in $90 \%$. [ $\mathrm{R}_{\mathrm{f}}=0.3$ (15\% EtOAc/hexanes), yellow oil]. ${ }^{1} \mathbf{H}-$ NMR $\left(\mathrm{CDCl}_{3}, 500 \mathrm{MHz}, \delta\right): 5.41(1 \mathrm{H}, \mathrm{dd}, J=15.3$ and $6.6 \mathrm{~Hz}, \mathrm{H}-5), 5.23(1 \mathrm{H}, \mathrm{m}, \mathrm{H}-$ 4), 3.59 (2H, m, H-1), $2.31(1 \mathrm{H}, \mathrm{m}, \mathrm{H}-6), 2.21\left(1 \mathrm{H}, \mathrm{m}, \mathrm{CH}_{2}\right), 2.05\left(2 \mathrm{H}, \mathrm{m}, \mathrm{CH}_{2}\right), 1.93$ $(1 \mathrm{H}, \mathrm{t}, J=2.6 \mathrm{~Hz}, \mathrm{H}-\mathrm{C} \equiv), 1.72(1 \mathrm{H}, \mathrm{m}, \mathrm{H}-2), 0.92(6 \mathrm{H}, \mathrm{d}, J=6.8 \mathrm{~Hz}) .{ }^{13} \mathbf{C}-\mathbf{N M R}$ $\left(\mathrm{CDCl}_{3}, 125 \mathrm{MHz}, \delta\right): 140.2(\mathrm{CH}), 123.9(\mathrm{CH}), 82.6(\mathrm{C} \equiv), 69.6(\mathrm{C} \equiv), 64.5\left(\mathrm{CH}_{2}\right)$, $39.9(\mathrm{CH}), 33.4\left(\mathrm{CH}_{2}\right), 30.9(\mathrm{CH}), 22.5\left(2 \mathrm{CH}_{3}\right), 19.6\left(\mathrm{CH}_{2}\right) . \mathbf{E M}-\mathrm{IQ}^{+}(\mathrm{m} / \mathrm{e}, \mathrm{I}): 167$ $\left(\mathrm{MH}^{+}, 7\right), 149\left(\mathrm{MH}^{+}-\mathrm{H}_{2} \mathrm{O}, 10\right)$. HRMS calcd. for $\mathrm{C}_{11} \mathrm{H}_{19} \mathrm{O}\left(\mathrm{MH}^{+}\right): 167.143590$, found 167.143558 .

Preparation of 5-methyl-2-(prop-2-ynyl)-4-hexen-1-ol. Following the above described procedure, 5-methyl-2-(prop-2-ynyl)-4-hexen-1-ol was obtained in 94\%. $\left[\mathrm{R}_{\mathrm{f}}=0.1\left(10 \%\right.\right.$ EtOAc/ hexanes), pale yellow oil]. 1H-NMR $\left(\mathrm{CDCl}_{3}, 300 \mathrm{MHz}, \delta\right)$ : 5.09 (1H, m, H-4), 3.62 (2H, m, H-1), 2.25 (2H, m, $\left.\underline{\mathrm{C}}_{2}-\mathrm{C} \equiv\right), 2.06$ (2H, m, H-3), 1.96 $(1 \mathrm{H}, \mathrm{t}, J=2.7 \mathrm{~Hz}, \mathrm{H}-\mathrm{C} \equiv), 1.68(3 \mathrm{H}, \mathrm{s}, \mathrm{Me}), 1.60(3 \mathrm{H}, \mathrm{s}, \mathrm{Me}) \cdot{ }^{13} \mathrm{C}-\mathbf{N M R}\left(\mathrm{CDCl}_{3}, 75\right.$ $\mathrm{MHz}, \delta): 133.7(\mathrm{C}), 121.7(\mathrm{CH}), 82.8(\mathrm{C} \equiv), 69.6(\mathrm{C} \equiv), 64.9\left(\mathrm{CH}_{2}\right), 40.5(\mathrm{CH}), 29.0$ $\left(\mathrm{CH}_{2}\right), 25.8\left(\mathrm{CH}_{3}\right), 19.9\left(\mathrm{CH}_{2}\right), 17.8\left(\mathrm{CH}_{3}\right)$. EM-IQ ${ }^{+}(\mathrm{m} / \mathrm{e}, \mathrm{I}): 153\left(\mathrm{MH}^{+}, 15\right), 135$ $\left(\mathrm{MH}^{+}-\mathrm{H}_{2} \mathrm{O}, 27\right)$. HRMS calcd. for $\mathrm{C}_{10} \mathrm{H}_{17} \mathrm{O}\left(\mathrm{MH}^{+}\right): 153.127940$, found 153.128027 .

Representative experiment of iodide preparation. Preparation of $(6 E, Z)$ 4-(iodomethyl)-6-octen-1-yne (5a). Triphenylphosphine (2.85 g, $10.87 \mathrm{mmol})$, imidazole $(1.85 \mathrm{~g}, 27.17 \mathrm{mmol})$ and iodine $(2.53 \mathrm{~g}, 9.96 \mathrm{mmol})$ were successively added to a solution of (4E, Z)-2-(prop-2-ynyl)-4-hexen-1-ol $(1.25 \mathrm{~g}, 9.06 \mathrm{mmol})$ in THF $(45 \mathrm{~mL})$ at $0{ }^{\circ} \mathrm{C}$. The resulting mixture was stirred at that temperature for 30 min, and then 30 minutes at rt. The mixture was poured into water $(30 \mathrm{~mL})$ and extracted with $\mathrm{Et}_{2} \mathrm{O}(2 \times 15 \mathrm{~mL})$. The combined organic phases were dried over $\mathrm{Na}_{2} \mathrm{SO}_{4}$, and concentrated under reduced pressure. The crude iodide was purified by flash chromatography on silica gel (hexanes) to yield $1.91 \mathrm{~g}$ of $\mathbf{5 a}$. $\left[85 \%, \mathrm{R}_{\mathrm{f}}=0.8\right.$ (10\% EtOAc/ hexanes), colorless oil]. ${ }^{1} \mathbf{H}-\mathbf{N M R}\left(\mathrm{CDCl}_{3}, 250 \mathrm{MHz}, \delta\right): 5.56(1 \mathrm{H}, \mathrm{m}$, H-6 or H-7), 5.31 (1H, m, H-6 or H-7), 3.33 (2H, m, $\left.-\mathrm{CH}_{2}-\mathrm{I}\right), 2.23$ (4H, m, H-3, H-5), $1.99(1 \mathrm{H}, \mathrm{t}, J=2.6 \mathrm{~Hz}, \mathrm{H}-\mathrm{C}), 1.65(3 \mathrm{H}, \mathrm{m}, \mathrm{H}-8), 1.55(1 \mathrm{H}, \mathrm{m}, \mathrm{H}-4) .{ }^{13} \mathbf{C}-\mathbf{N M R}$ $\left(\mathrm{CDCl}_{3}, 63 \mathrm{MHz}, \delta\right.$ ): 128.3 (trans $\mathrm{CH}$ ), 127.3 (trans $\mathrm{CH}$ ), 126.9 (cis $\mathrm{CH}$ ), 126.6 (cis $\mathrm{CH}), 81.4(\mathrm{C} \equiv), 70.1(\mathrm{C} \equiv), 38.9(\mathrm{CH}), 36.6\left(\mathrm{CH}_{2}\right), 23.4\left(\mathrm{CH}_{2}\right), 18.0\left(\mathrm{CH}_{3}\right), 13.2$ $\left(\mathrm{CH}_{2}\right)$.

Preparation of (6Z)-4-(iodomethyl)-6-nonen-1-yne (5b). Following the above described procedure, iodide $\mathbf{5 b}$ was obtained in $86 \%$. $\left[\mathrm{R}_{\mathrm{f}}=0.8(10 \% \mathrm{EtOAc} /\right.$ hexanes), colorless oil]. ${ }^{1} \mathbf{H}-\mathbf{N M R}\left(\mathrm{CDCl}_{3}, 250 \mathrm{MHz}, \delta\right): 5.49$ (1H, m, H-6 or H-7), $5.23\left(1 \mathrm{H}, \mathrm{m}, \mathrm{H}-6\right.$ or H-7), $3.34\left(2 \mathrm{H}, \mathrm{m}, \mathrm{CH}_{2}-\mathrm{I}\right), 2.35(2 \mathrm{H}, \mathrm{m}, \mathrm{H}-8), 2.27(2 \mathrm{H}, \mathrm{dd}, J=$ 6.7 and $2.6 \mathrm{~Hz}, \mathrm{H}-3), 2.17(2 \mathrm{H}, \mathrm{m}, \mathrm{H}-5), 2.00(1 \mathrm{H}, \mathrm{t}, J=2.6 \mathrm{~Hz}, \mathrm{H}-1), 1.55(1 \mathrm{H}, \mathrm{m}$, H-4), 0.96 (3H, t, J=7.5 Hz, H-9). ${ }^{13}$ C-NMR (CDCl $\left., 63 \mathrm{MHz}, \delta\right): 134.7(\mathrm{CH}), 125.0$ $(\mathrm{CH}), 81.4(\mathrm{C} \equiv), 70.2(\mathrm{C} \equiv), 39.3(\mathrm{CH}), 31.3\left(\mathrm{CH}_{2}\right), 23.5\left(\mathrm{CH}_{2}\right), 20.8\left(\mathrm{CH}_{2}\right), 14.1$ $\left(\mathrm{CH}_{3}\right), 13.0\left(\mathrm{CH}_{2}\right)$. EM-IQ ${ }^{+}(\mathrm{m} / \mathrm{e}, \mathrm{I}): 263\left(\mathrm{MH}^{+}, 0.1\right), 136\left(\mathrm{MH}^{+}-\mathrm{I}, 11\right), 121(15), 107$ (55). HRMS calcd for $\mathrm{C}_{10} \mathrm{H}_{16}\left(\mathrm{MH}^{+}-\mathrm{I}\right): 136.125201$, found 136.124674 .

Preparation of (6E)-4-(iodomethyl)-8-methyl-6-nonen-1-yne (5c). Following the above described procedure, iodide $5 \mathbf{c}$ was obtained in $85 \%$. $\left[\mathrm{R}_{\mathrm{f}}=0.9\right.$ (10\% EtOAc/hexanes), colorless oil]. ${ }^{1} \mathbf{H}-\mathbf{N M R}\left(\mathrm{CDCl}_{3}, 250 \mathrm{MHz}, \delta\right): 5.55(1 \mathrm{H}, \mathrm{dd}, J$ $=15.3$ and $6.6 \mathrm{~Hz}, \mathrm{H}-7), 5.26(1 \mathrm{H}, \mathrm{m}, \mathrm{H}-6), 3.36\left(2 \mathrm{H}, \mathrm{m},-\mathrm{C}_{2}-\mathrm{I}\right), 2.00(1 \mathrm{H}, \mathrm{t}, J=2.6$ 
Hz, H-C $\equiv), 1.50(1 \mathrm{H}, \mathrm{m}, \mathrm{H}-4), 0.89(6 \mathrm{H}, \mathrm{d}, J=6.8 \mathrm{~Hz}) .{ }^{13} \mathbf{C}-\mathbf{N M R}\left(\mathrm{CDCl}_{3}, 63 \mathrm{MHz}\right.$,

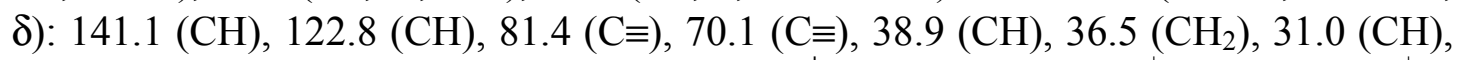
$23.3\left(\mathrm{CH}_{2}\right), 22.5\left(2 \mathrm{CH}_{3}\right), 13.1\left(\mathrm{CH}_{2}\right)$. EM-IQ ${ }^{+}(\mathrm{m} / \mathrm{e}, \mathrm{I}): 277\left(\mathrm{MH}^{+}, 2\right), 150\left(\mathrm{MH}^{+}-\mathrm{I}\right.$, 2).

Preparation of (4S, 6E)-4-(iodomethyl)-8-methyl-6-nonen-1-yne (5c-(S)). Following the above described procedure, iodide $\mathbf{5 c}-(\mathbf{S})$ was obtained in $75 \%$. $\left[\mathrm{R}_{\mathrm{f}}=\right.$ $0.9\left(10 \%\right.$ EtOAc/hexanes), colorless oil]. ${ }^{1} \mathbf{H}-\mathbf{N M R}\left(\mathrm{CDCl}_{3}, 250 \mathrm{MHz}, \delta\right): 5.55(1 \mathrm{H}$, $\mathrm{dd}, J=15.3$ and $6.6 \mathrm{~Hz}, \mathrm{H}-7), 5.26(1 \mathrm{H}, \mathrm{dt}, J=22.2$ and $7.2 \mathrm{~Hz}, \mathrm{H}-6), 3.35(2 \mathrm{H}, \mathrm{m},-$ $\left.\mathrm{C}_{H_{2}}-\mathrm{I}\right), 2.01(1 \mathrm{H}, \mathrm{t}, J=2.5 \mathrm{~Hz}, \mathrm{H}-\mathrm{C} \equiv), 1.50(1 \mathrm{H}, \mathrm{m}, \mathrm{H}-4), 0.89(6 \mathrm{H}, \mathrm{d}, J=6.7 \mathrm{~Hz})$. ${ }^{13} \mathbf{C}-\mathbf{N M R}\left(\mathrm{CDCl}_{3}, 63 \mathrm{MHz}, \delta\right): 141.1(\mathrm{CH}), 122.8(\mathrm{CH}), 81.4(\mathrm{C} \equiv), 70.1(\mathrm{C} \equiv), 38.9$ $(\mathrm{CH}), 36.5\left(\mathrm{CH}_{2}\right), 31.0(\mathrm{CH}), 23.3\left(\mathrm{CH}_{2}\right), 22.5\left(2 \mathrm{CH}_{3}\right), 13.1\left(\mathrm{CH}_{2}\right)$. EM-IQ ${ }^{+}(\mathrm{m} / \mathrm{e}, \mathrm{I})$ : $277\left(\mathrm{MH}^{+}, 4\right), 150\left(\mathrm{MH}^{+}-\mathrm{I}, 12\right)$. HRMS calcd for $\mathrm{C}_{11} \mathrm{H}_{17} \mathrm{I}\left(\mathrm{MH}^{+}\right): 277.045328$, found 277.046401 .

Preparation of 4-(iodomethyl)-7-methyloct-6-en-1-yne (5d). Following the above described procedure, iodide $\mathbf{5 d}$ was obtained in $98 \%$. $\left[\mathrm{R}_{\mathrm{f}}=0.8(10 \%\right.$ EtOAc/hexanes), colorless oil]. ${ }^{1} \mathbf{H}-\mathbf{R M N}\left(\mathrm{CDCl}_{3}, 250 \mathrm{MHz}, \delta\right): 5.00(1 \mathrm{H}, \mathrm{t}, J=6.8$ $\mathrm{Hz}, \mathrm{H}-6), 3.29\left(2 \mathrm{H}, \mathrm{m},-\mathrm{CH}_{2}-\mathrm{I}\right), 2.23(2 \mathrm{H}, \mathrm{m}, \mathrm{H}-3), 2.05(2 \mathrm{H}, \mathrm{dd}, J=12.3$ and $6.8 \mathrm{~Hz}$, $\mathrm{H}-5), 1.95(1 \mathrm{H}, \mathrm{t}, J=2.6 \mathrm{~Hz}, \mathrm{H}-\mathrm{C} \equiv), 1.65(3 \mathrm{H}, \mathrm{s}, \mathrm{MeC}=), 1.59(3 \mathrm{H}, \mathrm{s}, \mathrm{MeC}=), 1.51$ (1H, m,H-4). ${ }^{13} \mathbf{C}-\mathbf{N M R}\left(\mathrm{CDCl}_{3}, 63 \mathrm{MHz}, \delta\right): 134.4(\mathrm{C}), 120.8(\mathrm{CH}), 81.5(\mathrm{C} \equiv), 70.05$ $(\mathrm{C} \equiv), 39.6(\mathrm{CH}), 32.15\left(\mathrm{CH}_{2}\right), 25.8\left(\mathrm{CH}_{3}\right), 23.5\left(\mathrm{CH}_{2}\right), 18.1\left(\mathrm{CH}_{3}\right), 13.2\left(\mathrm{CH}_{2}\right)$. EMIQ $^{+}(\mathrm{m} / \mathrm{e}, \mathrm{I}): 263\left(\mathrm{MH}^{+}, 0.2\right), 136\left(\mathrm{MH}^{+}-\mathrm{I}, 2\right)$. HRMS calcd for $\mathrm{C}_{10} \mathrm{H}_{16} \mathrm{I}\left(\mathrm{MH}^{+}\right)$: 263.029678, found 263.030988.

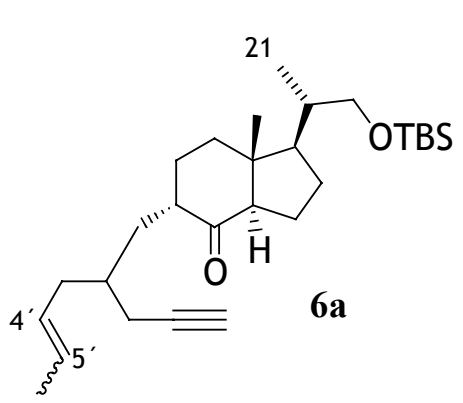

Representative ketone alkylation. Preparation of ketone 6a. To a solution of KHMDS $(0.5 \mathrm{M}$ in toluene, $9.3 \mathrm{~mL}, 4.6 \mathrm{mmol})$ in DMF $(4 \mathrm{~mL})$ cooled at $-78^{\circ} \mathrm{C}$ was slowly added a solution of ketone $3(0.50 \mathrm{~g}, 1.54 \mathrm{mmol})$ in DMF $(3 \mathrm{~mL})$. The resulting mixture was stirred for 0.5 $\mathrm{h}$, and a solution of iodide $\mathbf{5 a}(1.15 \mathrm{~g}, 4.64 \mathrm{mmol})$ in DMF $(2 \mathrm{~mL})$ was added. After $2 \mathrm{~h}$, a saturated solution of $\mathrm{NH}_{4} \mathrm{Cl}(4 \mathrm{~mL})$ was added and the aqueous layer was extracted with $\mathrm{Et}_{2} \mathrm{O}(2 \times 4 \mathrm{~mL})$. The combined organic extracts were washed with brine, dried over $\mathrm{Na}_{2} \mathrm{SO}_{4}$, and concentrated under reduced pressure to give a residue that was flash chromatographed on silica gel $(2 \%$ EtOAc/hexanes) to afford $540 \mathrm{mg}$ of $\mathbf{6 a}$. [79\%, $\mathrm{R}_{\mathrm{f}}=0.5(10 \% \mathrm{EtOAc} / \mathrm{hexanes})$, pale yellow oil]. ${ }^{1} \mathbf{H}-\mathbf{N M R}\left(\mathrm{CDCl}_{3}, 300 \mathrm{MHz}, \delta\right): 5.46\left(1 \mathrm{H}, \mathrm{m}, \mathrm{H}-4{ }^{\prime}\right.$ or $\left.\mathrm{H}-5^{\prime}\right), 5.30(1 \mathrm{H}, \mathrm{m}$, $\mathrm{H}-4^{\prime}$ or $\left.\mathrm{H}-5^{\prime}\right), 3.56\left(1 \mathrm{H}, \mathrm{dd}, J=9.5\right.$ and $\left.2.6 \mathrm{~Hz},-\mathrm{CH}_{\mathrm{a}}-\mathrm{OTBS}\right), 3.33(1 \mathrm{H}, \mathrm{dd}, J=9.5$ and $\left.5.9 \mathrm{~Hz},-\mathrm{CH}_{\mathrm{b}}-\mathrm{OTBS}\right), 1.94(1 \mathrm{H}, \mathrm{t}, J=2.6 \mathrm{~Hz}, \mathrm{H}-\mathrm{C} \equiv), 1.02(3 \mathrm{H}, \mathrm{d}, J=6.2 \mathrm{~Hz}$, Me-21), 0.90 (9H, s, $\left.{ }^{\mathrm{t}} \mathrm{Bu}\right), 0.65$ (3H, s, Me-18), 0.04 (6H, s, Me $\left.{ }_{2} \mathrm{Si}\right) .{ }^{13} \mathrm{C}-\mathbf{N M R}$ $\left(\mathrm{CDCl}_{3}, 75 \mathrm{MHz}, \delta\right): 214.8(\mathrm{CO}), 128.2(\mathrm{CH}), 127.5(\mathrm{CH}), 82.2(\mathrm{C} \equiv), 69.7(\mathrm{C} \equiv), 67.6$ $\left(\mathrm{CH}_{2}\right), 57.7(\mathrm{CH}), 53.3(\mathrm{CH}), 50.3(\mathrm{C}), 47.3(\mathrm{CH}), 38.7(\mathrm{CH}), 36.5\left(\mathrm{CH}_{2}\right), 35.9\left(\mathrm{CH}_{2}\right)$, $35.4\left(\mathrm{CH}_{2}\right), 35.0(\mathrm{CH}), 29.3\left(\mathrm{CH}_{2}\right), 27.1\left(\mathrm{CH}_{2}\right), 26.0\left(3 \mathrm{CH}_{3}\right), 21.4\left(\mathrm{CH}_{2}\right), 19.2\left(\mathrm{CH}_{2}\right)$, $18.5(\mathrm{C}), 18.0\left(\mathrm{CH}_{3}\right), 17.1\left(\mathrm{CH}_{3}\right), 12.9\left(\mathrm{CH}_{3}\right),-5.2\left(\mathrm{CH}_{3}\right),-5.3\left(\mathrm{CH}_{3}\right)$. EM-IQ ${ }^{+}(\mathrm{m} / \mathrm{e}$, I): $445\left(\mathrm{MH}^{+}, 29\right), 314\left(\mathrm{MH}^{+}-\mathrm{OTBS}, 15\right), 312\left(\mathrm{MH}^{+}-\mathrm{TBS}-\mathrm{H}_{2} \mathrm{O}, 5\right), 296\left(\mathrm{MH}^{+}-\mathrm{OTBS}-\right.$ $\left.\mathrm{H}_{2} \mathrm{O}, 17\right), 294\left(\mathrm{MH}^{+}-\mathrm{TBS}-\mathrm{H}_{2} \mathrm{O}-\mathrm{H}_{2} \mathrm{O}, 2\right)$. HRMS calcd for $\mathrm{C}_{28} \mathrm{H}_{49} \mathrm{O}_{2} \mathrm{Si}\left(\mathrm{MH}^{+}\right)$: 445.350185, found 445.350356. 


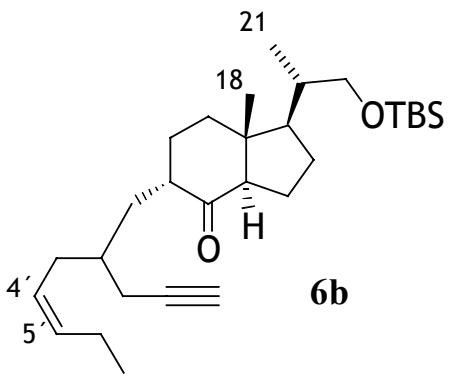

Preparation of ketone 6b. Following the above described procedure, ketone $\mathbf{6 b}$ was obtained in $35 \%$. $\left[\mathrm{R}_{\mathrm{f}}\right.$ $=0.2(5 \%$ EtOAc/hexanes $)$, pale yellow oil]. ${ }^{1} \mathbf{H}-\mathbf{N M R}$ $\left(\mathrm{CDCl}_{3}, 250 \mathrm{MHz}, \delta\right): 5.43\left(1 \mathrm{H}, \mathrm{m}, \mathrm{H}-4^{\prime}\right.$ or $\left.\mathrm{H}-5^{\prime}\right), 5.19$ $\left(1 \mathrm{H}, \mathrm{m}, \mathrm{H}-4^{\prime}\right.$ or $\left.\mathrm{H}-5^{\prime}\right), 3.54(1 \mathrm{H}, \mathrm{dd}, J=9.7$ and $2.3 \mathrm{~Hz}$, $\left.-\mathrm{CH}_{\mathrm{a}}-\mathrm{OTBS}\right), 3.29\left(1 \mathrm{H}, \mathrm{dd}, J=9.6\right.$ and $6.2 \mathrm{~Hz},-\mathrm{CH}_{\mathrm{b}^{-}}$ OTBS), 0.87 (9H, s, $\left.{ }^{\mathrm{t}} \mathrm{Bu}\right), 0.62(3 \mathrm{H}, \mathrm{s}, \mathrm{Me}-18), 0.01$ $\left(6 \mathrm{H}, \mathrm{s}, \mathrm{Me}_{2} \mathrm{Si}\right) \cdot{ }^{13} \mathrm{C}-\mathrm{NMR}\left(\mathrm{CDCl}_{3}, 63 \mathrm{MHz}, \delta\right): 214.9$ (CO), $133.9(\mathrm{CH}), 126.1(\mathrm{CH}), 82.0(\mathrm{C} \equiv), 69.7(\mathrm{C} \equiv), 67.5\left(\mathrm{CH}_{2}\right), 57.6(\mathrm{CH}), 53.2$ $(\mathrm{CH}), 50.2(\mathrm{C}), 47.6(\mathrm{CH}), 38.6(\mathrm{CH}), 36.3\left(\mathrm{CH}_{2}\right), 35.3\left(\mathrm{CH}_{2}\right), 35.1(\mathrm{CH}), 31.05$ $\left(\mathrm{CH}_{2}\right), 29.2\left(\mathrm{CH}_{2}\right), 27.0\left(\mathrm{CH}_{2}\right), 25.9\left(3 \mathrm{CH}_{3}\right), 21.3\left(\mathrm{CH}_{2}\right), 20.6\left(\mathrm{CH}_{2}\right), 19.0\left(\mathrm{CH}_{2}\right), 18.3$ (C), $17.0\left(\mathrm{CH}_{3}\right), 14.2\left(\mathrm{CH}_{3}\right), 12.8\left(\mathrm{CH}_{3}\right),-5.4\left(\mathrm{CH}_{3}\right),-5.5\left(\mathrm{CH}_{3}\right)$. EM-IQ ${ }^{+}(\mathrm{m} / \mathrm{e}, \mathrm{I})$ : $459\left(\mathrm{MH}^{+}, 23\right), 328\left(\mathrm{MH}^{+}\right.$-OTBS, 2), $310\left(\mathrm{MH}^{+}-\mathrm{OTBS}-\mathrm{H}_{2} \mathrm{O}, 3\right)$. HRMS calcd. for $\mathrm{C}_{29} \mathrm{H}_{51} \mathrm{O}_{2} \mathrm{Si}\left(\mathrm{MH}^{+}\right): 459.365835$, found 459.366409 .

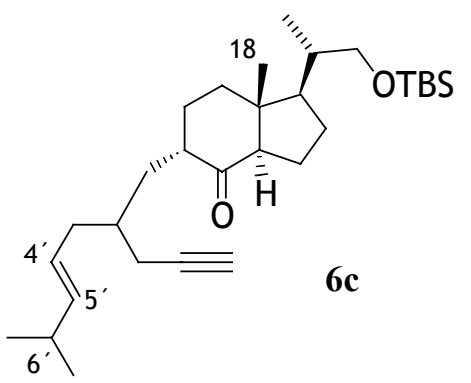

Preparation of ketone 6c. Following the above described procedure, ketone $\mathbf{6 c}$ was obtained in $78 \%$. $\left[\mathrm{R}_{\mathrm{f}}\right.$ $=0.3$ (5\% EtOAc/hexanes), pale yellow oil]. ${ }^{1} \mathbf{H}-\mathbf{N M R}$ $\left(\mathrm{CDCl}_{3}, 250 \mathrm{MHz}, \delta\right): 5.41(1 \mathrm{H}, \mathrm{dd}, J=15.3$ and $6.4 \mathrm{~Hz}$, H-5'), $5.22\left(1 \mathrm{H}, \mathrm{m}, \mathrm{H}-4^{\prime}\right), 3.51\left(1 \mathrm{H}, \mathrm{m},-\mathrm{CH}_{\mathrm{a}}-\mathrm{OTBS}\right)$, $3.31\left(1 \mathrm{H}, \mathrm{m},-\mathrm{CH}_{\mathrm{b}}-\mathrm{OTBS}\right), 2.57\left(1 \mathrm{H}, \mathrm{m}, \mathrm{H}-6^{\prime}\right), 0.87$ $\left(9 \mathrm{H}, \mathrm{s},{ }^{\mathrm{t}} \mathrm{Bu}\right), 0.62(3 \mathrm{H}, \mathrm{s}, \mathrm{Me}-18), 0.01\left(6 \mathrm{H}, \mathrm{s}, \mathrm{Me}_{2} \mathrm{Si}\right)$. ${ }^{13} \mathrm{C}-\mathrm{NMR}\left(\mathrm{CDCl}_{3}, 63 \mathrm{MHz}, \delta\right): 214.6(\mathrm{CO}), 140.4(\mathrm{CH})$, $124.1(\mathrm{CH}), 82.2(\mathrm{C} \equiv), 69.7(\mathrm{C} \equiv), 67.5\left(\mathrm{CH}_{2}\right), 57.6(\mathrm{CH}), 53.2(\mathrm{CH}), 50.3(\mathrm{C}), 47.2$ $(\mathrm{CH}), 38.5(\mathrm{CH}), 36.4\left(\mathrm{CH}_{2}\right), 35.7\left(\mathrm{CH}_{2}\right), 35.4\left(\mathrm{CH}_{2}\right), 34.9(\mathrm{CH}), 31.1(\mathrm{CH}), 29.3$ $\left(\mathrm{CH}_{2}\right), 27.0\left(\mathrm{CH}_{2}\right), 25.9\left(2 \mathrm{CH}_{3}\right), 22.6\left(3 \mathrm{CH}_{3}\right), 21.3\left(\mathrm{CH}_{2}\right), 19.0\left(\mathrm{CH}_{2}\right), 18.3(\mathrm{C}), 17.0$ $\left(\mathrm{CH}_{3}\right), 12.8\left(\mathrm{CH}_{3}\right),-5.4\left(2 \mathrm{CH}_{3}\right)$. EM-IQ ${ }^{+}(\mathrm{m} / \mathrm{e}, \mathrm{I}): 473\left(\mathrm{MH}^{+}, 100\right), 342\left(\mathrm{MH}^{+}-\right.$ OTBS, 6), 341 (28), 323 (12). HRMS calcd. for $\mathrm{C}_{30} \mathrm{H}_{53} \mathrm{O}_{2} \mathrm{Si}\left(\mathrm{MH}^{+}\right)$: 473.381485, found 473.383792 .

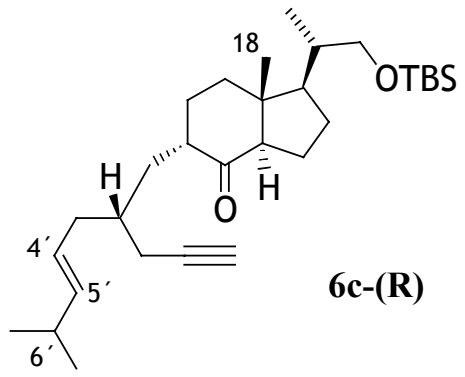

Preparation of ketone 6c-(R). Following the above described procedure, ketone $\mathbf{6 c - ( R )}$ was obtained in $70 \%$. $\left[\mathrm{R}_{\mathrm{f}}=0.3(5 \%\right.$ EtOAc/hexanes $)$, pale yellow oil $]$. ${ }^{1} \mathbf{H}-\mathbf{N M R}\left(\mathrm{CDCl}_{3}, 250 \mathrm{MHz}, \delta\right): 5.41\left(1 \mathrm{H}, \mathrm{m}, \mathrm{H}-5^{\prime}\right)$, $5.27\left(1 \mathrm{H}, \mathrm{m}, \mathrm{H}-4^{\prime}\right), 3.54\left(1 \mathrm{H}, \mathrm{m},-\mathrm{CH}_{\mathrm{a}}-\mathrm{OTBS}\right), 3.31$ $\left(1 \mathrm{H}, \mathrm{dd}, J=9.7\right.$ and $\left.6.1 \mathrm{~Hz},-\mathrm{CH}_{\mathrm{b}}-\mathrm{OTBS}\right), 0.87(9 \mathrm{H}, \mathrm{s}$, $\left.{ }^{\mathrm{t}} \mathrm{Bu}\right), 0.63(3 \mathrm{H}, \mathrm{s}, \mathrm{Me}-18), 0.01\left(6 \mathrm{H}, \mathrm{s}, \mathrm{Me}_{2} \mathrm{Si}\right) .{ }^{13} \mathrm{C}-$ NMR $\left(\mathrm{CDCl}_{3}, 63 \mathrm{MHz}, \delta\right): 214.8(\mathrm{CO}), 140.5(\mathrm{CH})$, $124.0(\mathrm{CH}), 82.1(\mathrm{C} \equiv), 69.5(\mathrm{C} \equiv), 67.5\left(\mathrm{CH}_{2}\right), 57.6(\mathrm{CH}), 53.2(\mathrm{CH}), 50.2(\mathrm{C}), 47.4$ $(\mathrm{CH}), 38.5(\mathrm{CH}), 36.3\left(\mathrm{CH}_{2}\right), 35.6\left(\mathrm{CH}_{2}\right), 35.4\left(\mathrm{CH}_{2}\right), 34.9(\mathrm{CH}), 31.0(\mathrm{CH}), 29.2$ $\left(\mathrm{CH}_{2}\right), 27.0\left(\mathrm{CH}_{2}\right), 25.9\left(2 \mathrm{CH}_{3}\right), 22.6\left(3 \mathrm{CH}_{3}\right), 21.3\left(\mathrm{CH}_{2}\right), 19.0\left(\mathrm{CH}_{2}\right), 18.3(\mathrm{C}), 17.0$ $\left(\mathrm{CH}_{3}\right), 12.8\left(\mathrm{CH}_{3}\right),-5.4\left(2 \mathrm{CH}_{3}\right) . \mathbf{E M}-\mathrm{IQ}^{+}(\mathrm{m} / \mathrm{e}, \mathrm{I}): 473\left(\mathrm{MH}^{+}, 13\right), 342\left(\mathrm{MH}^{+}-\mathrm{OTBS}\right.$, 7), 341 (28), 323 (33). HRMS calcd. for $\mathrm{C}_{30} \mathrm{H}_{53} \mathrm{O}_{2} \mathrm{Si}\left(\mathrm{MH}^{+}\right)$: 473.381485, found 473.382693 .

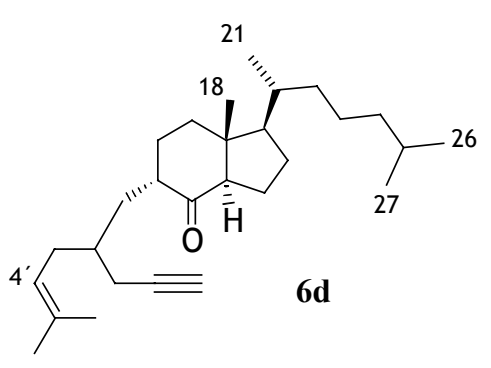

Preparation of ketone 6d. Following the above described procedure, ketone $6 \mathbf{d}$ was obtained in $69 \%$. [ $\mathrm{R}_{\mathrm{f}}$ $=0.6(10 \%$ EtOAc/hexanes $)$, pale yellow oil]. ${ }^{1} \mathbf{H}-\mathbf{N M R}$ $\left(\mathrm{CDCl}_{3}, 300 \mathrm{MHz}, \delta\right): 5.03\left(1 \mathrm{H}, \mathrm{t}, J=6.8 \mathrm{~Hz}, \mathrm{H}-4^{\prime}\right)$, $1.70\left(6 \mathrm{H}, \mathrm{s}, \mathrm{Me}_{2} \mathrm{C}=\right), 1.14(3 \mathrm{H}, \mathrm{d}, J=5.7 \mathrm{~Hz}, \mathrm{Me}-21)$, $0.87(6 \mathrm{H}, \mathrm{d}, J=6.6 \mathrm{~Hz}, \mathrm{Me}-26, \mathrm{Me}-27), 0.64(3 \mathrm{H}, \mathrm{s}$, Me-18). ${ }^{13}$ C-NMR $\left(\mathrm{CDCl}_{3}, 75 \mathrm{MHz}, \delta\right): 215.1$ (CO), $133.6(\mathrm{C}), 121.9(\mathrm{CH}), 82.3(\mathrm{C} \equiv), 69.5(\mathrm{C} \equiv), 57.8(\mathrm{CH})$, 
$56.8(\mathrm{CH}), 50.2(\mathrm{C}), 47.2(\mathrm{CH}), 39.3\left(\mathrm{CH}_{2}\right), 36.0\left(\mathrm{CH}_{2}\right), 35.9\left(\mathrm{CH}_{2}\right), 35.5(\mathrm{CH}), 35.4$ $\left(\mathrm{CH}_{2}\right), 31.9\left(\mathrm{CH}_{2}\right), 29.3\left(\mathrm{CH}_{2}\right), 27.9(\mathrm{CH}), 27.5\left(\mathrm{CH}_{2}\right), 25.8(\mathrm{CH}), 23.7\left(\mathrm{CH}_{2}\right), 22.7$ $\left(\mathrm{CH}_{3}\right), 22.5\left(\mathrm{CH}_{3}\right), 21.3\left(\mathrm{CH}_{2}\right), 18.9\left(\mathrm{CH}_{2}\right), 18.6\left(\mathrm{CH}_{3}\right), 17.9\left(\mathrm{CH}_{3}\right), 12.6\left(\mathrm{CH}_{3}\right) . \mathbf{E M}-$ IQ $^{+}$(m/e, I): $399\left(\mathrm{MH}^{+}, 94\right), 381\left(\mathrm{MH}^{+}-\mathrm{H}_{2} \mathrm{O}, 52\right)$. HRMS calcd for $\mathrm{C}_{28} \mathrm{H}_{47} \mathrm{O}\left(\mathrm{MH}^{+}\right)$: 399.362692, found 399.361999.

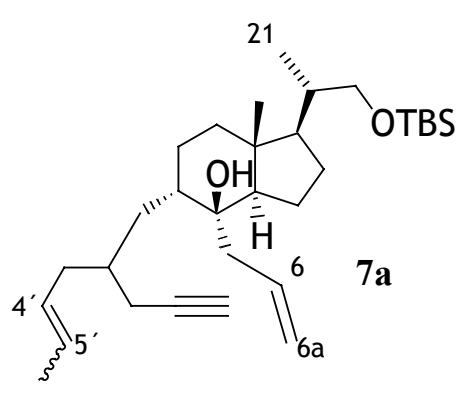

Representative dienyne preparation. Preparation of dienyne 7a. Allyl magnesium bromide (1M in THF, 4.7 $\mathrm{mL}, 4.7 \mathrm{mmol})$ was added dropwise to a solution of ketone 6a $(520 \mathrm{mg}, 1.17 \mathrm{mmol})$ in THF $(10 \mathrm{~mL})$ at -78 ${ }^{\circ} \mathrm{C}$. After $2 \mathrm{~h}$, a saturated solution of $\mathrm{NH}_{4} \mathrm{Cl}(15 \mathrm{~mL})$ was added, and the resulting mixture was extracted with $\mathrm{Et}_{2} \mathrm{O}(3 \times 10 \mathrm{~mL})$. The combined organic extracts were washed with brine, dried over $\mathrm{Na}_{2} \mathrm{SO}_{4}$, and concentrated under reduced pressure. The crude was purified by flash chromatography on silica gel (3\% EtOAc/hexanes) to yield $540 \mathrm{mg}$ of $7 \mathbf{a}$. [95\%, $\mathrm{R}_{\mathrm{f}}=$ 0.5 (10\% EtOAc/hexanes), pale yellow oil]. ${ }^{1} \mathbf{H}-\mathbf{N M R}\left(\mathrm{CDCl}_{3}, 500 \mathrm{MHz}, \delta\right): 5.90$ (1H, m, H-6), 5.39 (2H, m, H-4', H-5'), $5.13(2 \mathrm{H}, \mathrm{m}, \mathrm{H}-6 \mathrm{a}), 3.54\left(1 \mathrm{H}, \mathrm{m},-\mathrm{CH}_{\mathrm{a}}-\right.$ OTBS), 3.24 (1H, m, $-\mathrm{CH}_{\mathrm{b}}$-OTBS), $0.95(3 \mathrm{H}, \mathrm{d}, J=6.3 \mathrm{~Hz}, \mathrm{Me}-21), 0.87\left(9 \mathrm{H}, \mathrm{s},{ }^{\mathrm{t}} \mathrm{Bu}\right)$, $0.01\left(6 \mathrm{H}, \mathrm{s}, \mathrm{Me}_{2} \mathrm{Si}\right) .{ }^{13} \mathrm{C}-\mathrm{NMR}\left(\mathrm{CDCl}_{3}, 125 \mathrm{MHz}, \delta\right): 133.2(\mathrm{CH}), 128.3(\mathrm{CH}), 127.4$ $(\mathrm{CH}), 119.4\left(\mathrm{CH}_{2}\right), 83.3(\mathrm{C} \equiv), 76.0(\mathrm{C}), 67.3(\mathrm{C} \equiv), 67.7\left(\mathrm{CH}_{2}\right), 53.6(\mathrm{CH}), 51.1(\mathrm{CH})$, $43.4\left(\mathrm{CH}_{2}\right), 43.3(\mathrm{C}), 39.5(\mathrm{CH}), 38.5(\mathrm{CH}), 35.2(\mathrm{CH}), 34.8\left(\mathrm{CH}_{2}\right), 34.5\left(\mathrm{CH}_{2}\right), 31.2$ $\left(\mathrm{CH}_{2}\right), 26.7\left(\mathrm{CH}_{2}\right), 26.0\left(3 \mathrm{CH}_{3}\right), 23.8\left(\mathrm{CH}_{2}\right), 20.3\left(\mathrm{CH}_{2}\right), 20.2\left(\mathrm{CH}_{2}\right), 18.3(\mathrm{C}), 18.0$ $\left(\mathrm{CH}_{3}\right), 16.7\left(\mathrm{CH}_{3}\right), 13.5\left(\mathrm{CH}_{3}\right),-5.4\left(2 \mathrm{CH}_{3}\right) . \mathbf{E M}-\mathrm{IQ}^{+}(\mathrm{m} / \mathrm{e}, \mathrm{I}): 487\left(\mathrm{MH}^{+}, 19\right), 355$ (47), 337 (100). HRMS calcd. for $\mathrm{C}_{31} \mathrm{H}_{55} \mathrm{O}_{2} \mathrm{Si}\left(\mathrm{MH}^{+}\right): 487.397135$, found 487.396244 .

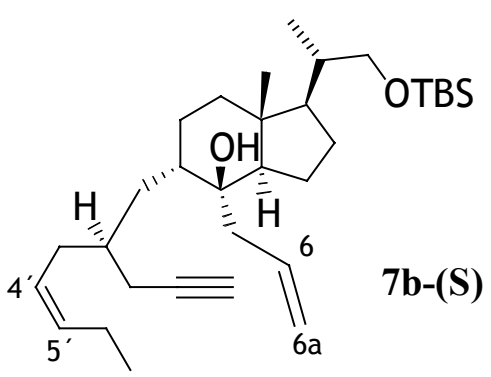

Preparation of dienyne $7 \mathrm{~b}$. Following the above described procedure, dienyne $\mathbf{7 b}-(\mathbf{S})$ and $\mathbf{7 b}-(\mathbf{R})$ were obtained from $\mathbf{6 b}$ in $70 \%$ in a $1: 1$ mixture that was separated by flash chromatography on silica gel.

7b-(S). $\left[\mathrm{R}_{\mathrm{f}}=0.42(10 \%\right.$ EtOAc/hexanes $)$, pale yellow oil]. ${ }^{1} \mathbf{H}-\mathbf{N M R}\left(\mathrm{CDCl}_{3}, 500 \mathrm{MHz}, \delta\right): 5.86(1 \mathrm{H}, \mathrm{m}, \mathrm{H}-$ 6), $5.45\left(1 \mathrm{H}, \mathrm{m}, \mathrm{H}-4^{\prime}\right.$ or $\left.\mathrm{H}-5^{\prime}\right), 5.28\left(1 \mathrm{H}, \mathrm{m}, \mathrm{H}-4^{\prime}\right.$ or $\mathrm{H}-$ $\left.5^{\prime}\right), 5.16(2 \mathrm{H}, \mathrm{m}, \mathrm{H}-6 \mathrm{a}), 3.56(1 \mathrm{H}, \mathrm{dd}, J=9.6$ and 3.3 $\left.\mathrm{Hz},-\mathrm{CH}_{a}-\mathrm{OTBS}\right), 3.26(1 \mathrm{H}, \mathrm{dd}, J=9.5$ and $7.2 \mathrm{~Hz}$, , $\mathrm{CH}_{b}$-OTBS), $0.88\left(9 \mathrm{H}, \mathrm{s},{ }^{\mathrm{t}} \mathrm{Bu}\right), 0.02\left(6 \mathrm{H}, \mathrm{s}, \mathrm{Me}_{2} \mathrm{Si}\right) .{ }^{13} \mathbf{C}-\mathbf{R M N}\left(\mathrm{CDCl}_{3}, 125 \mathrm{MHz}\right.$,

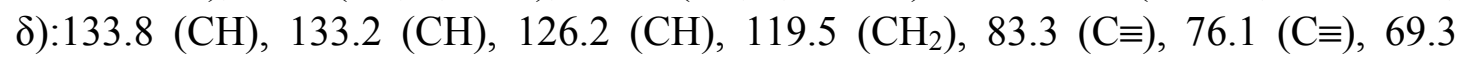
$\left(\mathrm{CH}_{2}\right), 67.7(\mathrm{C}), 53.7(\mathrm{CH}), 51.2(\mathrm{CH}), 43.5\left(\mathrm{CH}_{2}\right), 43.4(\mathrm{C}), 39.7(\mathrm{CH}), 38.5(\mathrm{CH})$, 35.6 (CH), 34.6 $\left(\mathrm{CH}_{2}\right), 31.6\left(\mathrm{CH}_{2}\right), 29.7\left(\mathrm{CH}_{2}\right), 26.7\left(\mathrm{CH}_{2}\right), 26.0\left(3 \mathrm{CH}_{3}\right), 23.8\left(\mathrm{CH}_{2}\right)$, $20.7\left(\mathrm{CH}_{2}\right), 20.5\left(\mathrm{CH}_{2}\right), 20.3\left(\mathrm{CH}_{2}\right), 18.4(\mathrm{C}), 16.7\left(\mathrm{CH}_{3}\right), 14.3\left(\mathrm{CH}_{3}\right), 13.5\left(\mathrm{CH}_{3}\right),-$ $5.3\left(\mathrm{CH}_{3}\right),-5.4\left(\mathrm{CH}_{3}\right)$. EM-IQ ${ }^{+}(\mathrm{m} / \mathrm{e}, \mathrm{I}): 501\left(\mathrm{MH}^{+}, 22\right), 370\left(\mathrm{MH}^{+}-\mathrm{OTBS}, 7\right), 352$ $\left(\mathrm{MH}^{+}\right.$- OTBS- $\left.\mathrm{H}_{2} \mathrm{O}, 31\right)$. HRMS calcd. for $\mathrm{C}_{32} \mathrm{H}_{57} \mathrm{O}_{2} \mathrm{Si}\left(\mathrm{MH}^{+}\right)$: 501.412785, found 501.413288 .

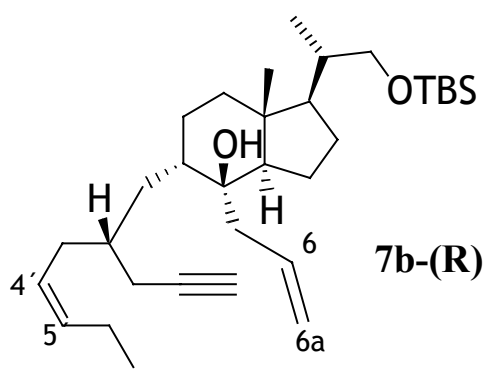

7b-(R). $\left[\mathrm{R}_{\mathrm{f}}=0.37(10 \%\right.$ EtOAc/hexanes), pale yellow oil]. ${ }^{1} \mathrm{H}-\mathrm{NMR}\left(\mathrm{CDCl}_{3}, 500 \mathrm{MHz}, \delta\right): 5.98(1 \mathrm{H}, \mathrm{m}, \mathrm{H}-$ 6), $5.46\left(1 \mathrm{H}, \mathrm{m}, \mathrm{H}-4^{\prime}\right.$ or $\left.\mathrm{H}-5^{\prime}\right), 5.29\left(1 \mathrm{H}, \mathrm{m}, \mathrm{H}-4^{\prime}\right.$ or H$\left.5^{\prime}\right), 5.15(2 \mathrm{H}, \mathrm{m}, \mathrm{H}-6 \mathrm{a}), 3.56(1 \mathrm{H}, \mathrm{dd}, J=9.6$ and 3.2 $\left.\mathrm{Hz},-\mathrm{CH}_{a}-\mathrm{OTBS}\right), 3.25(1 \mathrm{H}, \mathrm{dd}, J=9.5$ and $7.5 \mathrm{~Hz}$, , $\mathrm{CH}_{b}$-OTBS $), 0.88$ (9H, s, $\left.{ }^{t} \mathrm{Bu}\right), 0.02\left(6 \mathrm{H}, \mathrm{s}, \mathrm{Me}_{2} \mathrm{Si}\right)$. ${ }^{13} \mathbf{C}-\mathbf{R M N}\left(\mathrm{CDCl}_{3}, 125 \mathrm{MHz}, \delta\right): 133.6(\mathrm{CH}), 133.3$ 
$(\mathrm{CH}), 126.8(\mathrm{CH}), 119.7\left(\mathrm{CH}_{2}\right), 82.5(\mathrm{C} \equiv), 75.8(\mathrm{C} \equiv), 69.8\left(\mathrm{CH}_{2}\right), 67.7(\mathrm{C}), 53.7$ $(\mathrm{CH}), 51.2(\mathrm{CH}), 43.5\left(\mathrm{CH}_{2}\right), 43.3(\mathrm{C}), 39.8(\mathrm{CH}), 38.5(\mathrm{CH}), 34.8(\mathrm{CH}), 34.6\left(\mathrm{CH}_{2}\right)$, $32.2\left(\mathrm{CH}_{2}\right), 30.8\left(\mathrm{CH}_{2}\right), 26.7\left(\mathrm{CH}_{2}\right), 26.0\left(3 \mathrm{CH}_{3}\right), 21.1\left(\mathrm{CH}_{2}\right), 20.7\left(\mathrm{CH}_{2}\right), 20.35$ $\left(\mathrm{CH}_{2}\right), 20.3\left(\mathrm{CH}_{2}\right), 18.4(\mathrm{C}), 16.7\left(\mathrm{CH}_{3}\right), 14.3\left(\mathrm{CH}_{3}\right), 13.5\left(\mathrm{CH}_{3}\right),-5.3\left(\mathrm{CH}_{3}\right),-5.4$ $\left(\mathrm{CH}_{3}\right)$. EM-IQ ${ }^{+}$(m/e, I): $501\left(\mathrm{MH}^{+}, 0.05\right), 352\left(\mathrm{MH}^{+}-\mathrm{OTBS}_{-} \mathrm{H}_{2} \mathrm{O}, 2\right)$. HRMS calcd. for $\mathrm{C}_{32} \mathrm{H}_{57} \mathrm{O}_{2} \mathrm{Si}\left(\mathrm{MH}^{+}\right)$: 501.412785, found 501.413082.

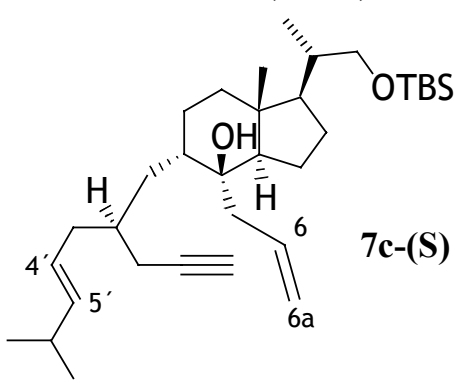

Preparation of dienyne 7c. Following the above

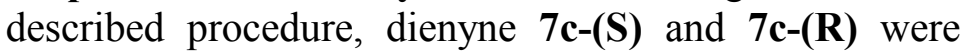
obtained from $\mathbf{6 c}$ in $80 \%$ in a $3: 1$ mixture that was separated by flash chromatography on silica gel.

7c-(S). $\left[\mathrm{R}_{\mathrm{f}}=0.35\right.$ (5\% EtOAc/hexanes), pale yellow oil]. ${ }^{1}$ H-NMR $\left(\mathrm{CDCl}_{3}, 300 \mathrm{MHz}, \delta\right): 5.88(1 \mathrm{H}, \mathrm{m}, \mathrm{H}-6), 5.48$ $\left(1 \mathrm{H}, \mathrm{m}, \mathrm{H}-5^{\prime}\right), 5.32\left(1 \mathrm{H}, \mathrm{m}, \mathrm{H}-4^{\prime}\right), 5.20(2 \mathrm{H}, \mathrm{m}, \mathrm{H}-6 \mathrm{a})$, $3.60\left(1 \mathrm{H}, \mathrm{m},-\mathrm{CH}_{a}\right.$-OTBS $), 3.29\left(1 \mathrm{H}, \mathrm{m},-\mathrm{CH}_{\mathrm{b}}-\mathrm{OTBS}\right)$, $1.95(1 \mathrm{H}, \mathrm{t}, J=2.5 \mathrm{~Hz}, \mathrm{H}-\mathrm{C} \equiv), 0.86\left(9 \mathrm{H}, \mathrm{s},{ }^{\mathrm{t}} \mathrm{Bu}\right), 0.06$ $\left(6 \mathrm{H}, \mathrm{s}, \mathrm{Me}_{2} \mathrm{Si}\right) .{ }^{13} \mathbf{C}-\mathbf{R M N}\left(\mathrm{CDCl}_{3}, 75 \mathrm{MHz}, \delta\right): 140.3(\mathrm{CH}), 133.2(\mathrm{CH}), 123.9(\mathrm{CH})$, $119.5\left(\mathrm{CH}_{2}\right), 83.5(\mathrm{C} \equiv), 69.3(\mathrm{C} \equiv), 76.0(\mathrm{C}), 67.7\left(\mathrm{CH}_{2}\right), 53.6(\mathrm{CH}), 51.1(\mathrm{CH}), 43.5$ $\left(\mathrm{CH}_{2}\right), 43.3(\mathrm{C}), 39.5(\mathrm{CH}), 38.5(\mathrm{CH}), 35.3(\mathrm{CH}), 34.9\left(\mathrm{CH}_{2}\right), 34.5\left(\mathrm{CH}_{2}\right), 31.3$ $\left(\mathrm{CH}_{2}\right), 31.1(\mathrm{CH}), 26.7\left(\mathrm{CH}_{2}\right), 26.0\left(2 \mathrm{CH}_{3}\right), 23.7\left(\mathrm{CH}_{2}\right), 22.6\left(\mathrm{CH}_{3}\right), 20.4\left(\mathrm{CH}_{2}\right), 20.3$ $\left(\mathrm{CH}_{2}\right), 18.4(\mathrm{C}), 16.7\left(3 \mathrm{CH}_{3}\right), 13.5\left(\mathrm{CH}_{3}\right),-5.4\left(2 \mathrm{CH}_{3}\right)$. EM-IQ ${ }^{+}(\mathrm{m} / \mathrm{e}, \mathrm{I}): 515\left(\mathrm{MH}^{+}\right.$, 22), $497\left(\mathrm{MH}^{+}-\mathrm{H}_{2} \mathrm{O}, 9\right), 384\left(\mathrm{MH}^{+}-\mathrm{OTBS}, 10\right), 383$ (41), 365 (100), 323(38). HRMS calcd. for $\mathrm{C}_{33} \mathrm{H}_{59} \mathrm{O}_{2} \mathrm{Si}\left(\mathrm{MH}^{+}\right)$: 515.428435 , found 515.428098.

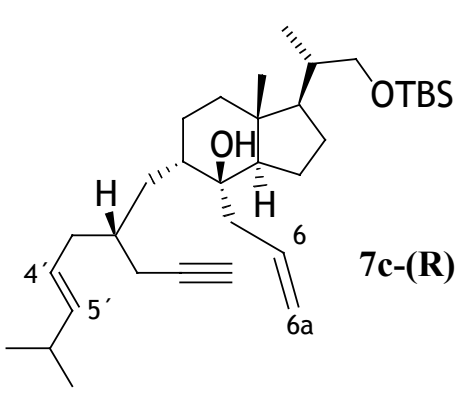

7c-(R). $\left[\mathrm{R}_{\mathrm{f}}=0.29\right.$ (10\% EtOAc/hexanes), pale yellow oil. ${ }^{1}$ H-NMR $\left(\mathrm{CDCl}_{3}, 300 \mathrm{MHz}, \delta\right): 5.86(1 \mathrm{H}, \mathrm{m}, \mathrm{H}-6), 5.44$ $\left(1 \mathrm{H}, \mathrm{m}, \mathrm{H}-5^{\prime}\right), 5.29\left(1 \mathrm{H}, \mathrm{m}, \mathrm{H}-4^{\prime}\right), 5.16(2 \mathrm{H}, \mathrm{m}, \mathrm{H}-6 \mathrm{a})$, $3.56\left(1 \mathrm{H}, \mathrm{dd}, J=3.4\right.$ and $\left.9.6 \mathrm{~Hz},-\mathrm{CH}_{\mathrm{a}}-\mathrm{OTBS}\right), 3.25(1 \mathrm{H}$, $\mathrm{dd}, J=7.3$ and $\left.9.6 \mathrm{~Hz},-\mathrm{CH}_{\mathrm{b}}-\mathrm{OTBS}\right), 1.95(1 \mathrm{H}, \mathrm{t}, J=2.6$ $\mathrm{Hz}, \mathrm{H}-\mathrm{C} \equiv), 0.89\left(9 \mathrm{H}, \mathrm{s},{ }^{\mathrm{t}} \mathrm{Bu}\right), 0.03\left(6 \mathrm{H}, \mathrm{s}, \mathrm{Me}_{2} \mathrm{Si}\right) .{ }^{13} \mathrm{C}-$ RMN $\left(\mathrm{CDCl}_{3}, 75 \mathrm{MHz}, \delta\right): 140.1(\mathrm{CH}), 133.3(\mathrm{CH})$, $124.8(\mathrm{CH}), 119.6\left(\mathrm{CH}_{2}\right), 82.6(\mathrm{C} \equiv), 75.8(\mathrm{C}), 69.7(\mathrm{C} \equiv)$, $67.7\left(\mathrm{CH}_{2}\right), 53.7(\mathrm{CH}), 51.2(\mathrm{CH}), 43.5\left(\mathrm{CH}_{2}\right), 43.3(\mathrm{C})$, $39.8(\mathrm{CH}), 38.4(\mathrm{CH}), 37.5\left(\mathrm{CH}_{2}\right), 34.7(\mathrm{CH}), 34.6\left(\mathrm{CH}_{2}\right), 31.0(\mathrm{CH}), 30.4\left(\mathrm{CH}_{2}\right)$, 26.6 $\left(\mathrm{CH}_{2}\right), 26.0\left(3 \mathrm{CH}_{3}\right), 22.7\left(2 \mathrm{CH}_{3}\right), 21.3\left(\mathrm{CH}_{2}\right), 20.3\left(\mathrm{CH}_{2}\right), 20.2\left(\mathrm{CH}_{2}\right), 18.4(\mathrm{C})$, $16.6\left(\mathrm{CH}_{3}\right), 13.5\left(\mathrm{CH}_{3}\right),-5.4\left(2 \mathrm{CH}_{3}\right)$. EM-IQ ${ }^{+}(\mathrm{m} / \mathrm{e}, \mathrm{I}): 515\left(\mathrm{MH}^{+}, 14\right), 384\left(\mathrm{MH}^{+}-\right.$ OTBS, 1), $364\left(\mathrm{MH}^{+}-\mathrm{TBS}-2 \mathrm{H}_{2} \mathrm{O}, 0.3\right)$. HRMS calcd. for $\mathrm{C}_{33} \mathrm{H}_{59} \mathrm{O}_{2} \mathrm{Si}\left(\mathrm{MH}^{+}\right)$: 515.428435, found 515.429374.

Preparation of dienyne $\mathbf{7 c - ( R )}$. Following the above described procedure, dienyne $7 \mathbf{c}-(\mathbf{R})$ was obtained from $\mathbf{6 c}-(\mathbf{R})$ in $80 \%$. [ $\mathrm{R}_{\mathrm{f}}=0.29(10 \%$ EtOAc/hexanes $)$, pale yellow oil].

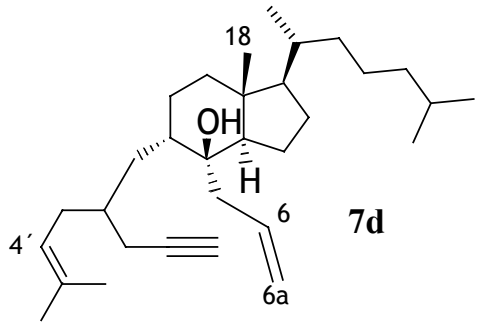

Preparation of dienyne 7d. Following the above described procedure, dienyne $\mathbf{7 d}$ was obtained in $80 \%$. $\left[\mathrm{R}_{\mathrm{f}}=0.5\right.$ (10\% EtOAc/hexanes), pale yellow oil $] .{ }^{\mathbf{1}} \mathbf{H}-$ NMR $\left(\mathrm{CDCl}_{3}, 300 \mathrm{MHz}, \delta\right): 5.83(1 \mathrm{H}, \mathrm{m}, \mathrm{H}-6), 5.03$ $\left(3 \mathrm{H}, \mathrm{m}, \mathrm{H}-6 \mathrm{a}, \mathrm{H}-4^{\prime}\right), 0.88$ (3H, s, Me-18). ${ }^{13} \mathbf{C}-\mathbf{N M R}$ $\left(\mathrm{CDCl}_{3}, 75 \mathrm{MHz}, \delta\right): 133.3(\mathrm{C}), 133.2(\mathrm{CH}), 121.1(\mathrm{CH})$, $119.3\left(\mathrm{CH}_{2}\right), 83.5(\mathrm{C} \equiv), 76.0(\mathrm{C}), 69.2(\mathrm{C} \equiv), 57.1(\mathrm{CH})$, $51.3(\mathrm{CH}), 43.3(\mathrm{C}), 43.4\left(\mathrm{CH}_{2}\right), 39.6(\mathrm{CH}), 39.5\left(\mathrm{CH}_{2}\right)$, $35.85(\mathrm{CH}), 35.8\left(\mathrm{CH}_{2}\right), 35.2(\mathrm{CH}), 34.6\left(\mathrm{CH}_{2}\right), 33.0\left(\mathrm{CH}_{2}\right), 31.5\left(\mathrm{CH}_{2}\right), 30.25\left(\mathrm{CH}_{2}\right)$, $28.0(\mathrm{CH}), 27.2\left(\mathrm{CH}_{2}\right), 25.8\left(\mathrm{CH}_{3}\right), 23.7\left(\mathrm{CH}_{2}\right), 22.8\left(\mathrm{CH}_{3}\right), 22.5\left(\mathrm{CH}_{3}\right), 20.4\left(\mathrm{CH}_{2}\right)$, $20.0\left(\mathrm{CH}_{2}\right), 18.3\left(\mathrm{CH}_{3}\right), 17.8\left(\mathrm{CH}_{3}\right), 13.4\left(\mathrm{CH}_{3}\right) . \mathbf{E M}-\mathrm{IQ}^{+}(\mathrm{m} / \mathrm{e}, \mathrm{I}): 441\left(\mathrm{MH}^{+}, 44\right)$, 
$423\left(\mathrm{MH}^{+}-\mathrm{H}_{2} \mathrm{O}, 100\right)$. HRMS calcd. for $\mathrm{C}_{31} \mathrm{H}_{53} \mathrm{O}\left(\mathrm{MH}^{+}\right)$: 441.409642, found 441.409447.

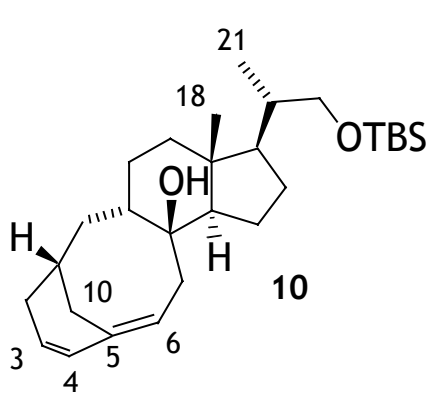

Representative dienyne metathesis. Preparation of 10. Grubbs's catalyst 11a (34 $\mathrm{mg}, 0.04 \mathrm{mmol}$ ) was added to a solution of dienyne $7 \mathbf{c}-(\mathbf{R})(211 \mathrm{mg}, 0.41 \mathrm{mmol})$ in $\mathrm{CH}_{2} \mathrm{Cl}_{2}$ $(80 \mathrm{~mL})$. The reaction mixture was refluxed for $3 \mathrm{~h}$, allowed to come to rt and concentrated under reduced pressure. The crude residue was purified by flash chromatography on alumine oxide $\left(8 \% \mathrm{H}_{2} \mathrm{O}\right)$ to yield $146 \mathrm{mg}$ of $\mathbf{1 0}$. [80\%, $\mathrm{R}_{\mathrm{f}}=$ 0.27 (10\% EtOAc/hexanes), yellow oil] ${ }^{1} \mathbf{H}-\mathbf{R M N}\left(\mathrm{CDCl}_{3}\right.$, $500 \mathrm{MHz}, \delta): 6.18(1 \mathrm{H}, \mathrm{d}, J=10.1 \mathrm{~Hz}, \mathrm{H}-4), 5.43(1 \mathrm{H}, \mathrm{m}$, $\mathrm{H}-3), 5.34(1 \mathrm{H}, \mathrm{t}, J=8.1 \mathrm{~Hz}, \mathrm{H}-6), 3.61\left(1 \mathrm{H}, \mathrm{dd}, J=9.6\right.$ and $\left.3.5 \mathrm{~Hz},-\mathrm{CH}_{\mathrm{a}}-\mathrm{OTBS}\right)$, $3.20\left(1 \mathrm{H}, \mathrm{m},-\mathrm{CH}_{\mathrm{b}}-\mathrm{OTBS}\right), 2.87(1 \mathrm{H}, \mathrm{dd}, J=11.4$ and $3.9 \mathrm{~Hz}, \mathrm{H}-10), 2.01(1 \mathrm{H}, \mathrm{d}, J=$ $11.4 \mathrm{~Hz}, \mathrm{H}-10), 0.91\left(9 \mathrm{H}, \mathrm{s},{ }^{\mathrm{t}} \mathrm{Bu}\right), 0.05\left(3 \mathrm{H}, \mathrm{s}, \mathrm{Me}_{2} \mathrm{Si}\right) .{ }^{13} \mathbf{C}-\mathbf{N M R}\left(\mathrm{CDCl}_{3}, 125 \mathrm{MHz}\right.$, $\delta): 139.7(\mathrm{C}), 130.5(\mathrm{CH}), 125.8(\mathrm{CH}), 119.1(\mathrm{CH}), 81.2(\mathrm{C}), 67.8\left(\mathrm{CH}_{2}\right), 53.8(\mathrm{CH})$, $48.5(\mathrm{CH}), 45.2(\mathrm{CH}), 43.7(\mathrm{C}), 40.5\left(\mathrm{CH}_{2}\right), 38.6(\mathrm{CH}), 35.6\left(\mathrm{CH}_{2}\right), 35.1\left(\mathrm{CH}_{2}\right), 34.9$ (CH), $33.3\left(\mathrm{CH}_{2}\right), 30.4\left(\mathrm{CH}_{2}\right), 29.9\left(\mathrm{CH}_{2}\right), 26.5\left(\mathrm{CH}_{2}\right), 26.0\left(3 \mathrm{CH}_{3}\right), 20.6\left(\mathrm{CH}_{2}\right), 18.4$ (C), $16.4\left(\mathrm{CH}_{3}\right), 14.0\left(\mathrm{CH}_{3}\right),-5.3\left(2 \mathrm{CH}_{3}\right) . \mathbf{E M}-\mathrm{IQ}^{+}(\mathrm{m} / \mathrm{e}, \mathrm{I}): 445\left(\mathrm{MH}^{+}, 4\right), 313(4)$, 295 (83). HRMS calcd for $\mathrm{C}_{28} \mathrm{H}_{49} \mathrm{O}_{2} \mathrm{Si}\left(\mathrm{MH}^{+}\right)$: 445.350185, found 445.351417. 